\title{
UNIQUE CONTINUATION FOR $\Delta+v$ AND THE C. FEFFERMAN-PHONG CLASS
}

\author{
SAGUN CHANILLO AND ERIC SAWYER
}

\begin{abstract}
We show that the strong unique continuation property holds for the inequality $|\Delta u| \leq|v||u|$, where the potential $v(x)$ satisfies the C. FeffermanPhong condition in a certain range of $p$ values. We also deal with the situation of $u(x)$ vanishing at infinity. These are all consequences of appropriate Carleman inequalities.
\end{abstract}

\section{INTRODUCTION}

Our aim is to prove local and global unique continuation theorems for the differential operator $Q(D)=\Delta+\sum_{j=1}^{n} a_{j} \partial / \partial x_{j}+\gamma, a_{i}, \gamma \in \mathbf{C}$. We assume that for $x \in \Omega \subset \mathbf{R}^{n}, n \geq 3$,

$$
|Q(D) u(x)| \leq v(x)|u(x)| .
$$

The problem is to find conditions on $v(x)$ that guarantee that any $u$ satisfying (1.1), and vanishing appropriately at a point, possibly infinity, must vanish identically. In [JK, $\mathrm{ST}_{1}$ and $\mathrm{KRS}$ ], unique continuation is proved for $v$ locally in $L^{n / 2}$ or locally small in weak $L^{n / 2}$, and in addition it is shown that uniqueness can fail for $L^{p}, p<n / 2$. In this paper we consider instead the class of potentials studied by C. Fefferman and D. Phong [F]. We say $v \in F_{p}$ if

$$
\|v\|_{F_{p}}=\sup _{Q}|Q|^{2 / n}\left(\frac{1}{|Q|} \int_{Q}|v|^{p}\right)^{1 / p}<\infty .
$$

We have $F_{q} \subset F_{p}$ if $p<q$ and $F_{n / 2}=L^{n / 2}$. We will prove unique continuation for $v$ that are locally small in $F_{p}$ with $p>(n-1) / 2$. Note that if $v \in$ weak $L^{n / 2}$ then $v \in F_{p}$ for all $p<n / 2$. On the other hand if $v(x)=f(x /|x|)|x|^{-2}, f \in L^{p}\left(S^{n-1}\right),(n-1) / 2<p<n / 2$, then $v$ need not be in weak $L^{n / 2}$, but $v \in F_{p}$. Thus our unique continuation results improve on the corresponding results in [JK, $\mathrm{ST}_{1}$ and KRS] by permitting potentials in $L_{\text {loc }}^{p}, p>(n-1) / 2$, but subject to the growth condition of Fefferman and

Received by the editors June 27, 1988.

1980 Mathematics Subject Classification (1985 Revision). Primary 35J10, 42B20, 35P10, 35B60; Secondary 35J05, 35P20.

The first author was supported in part by NSF grant DMS-8601119, and the second author by NSERC grant A5149. 
Phong. We do not know if unique continuation holds for $v$ that are locally small in $F_{p}$ with $1 \leq p \leq(n-1) / 2$. We remark that for $p=1$, the condition $F_{1}$ is weaker than the Kato condition,

$$
\lim _{\delta \rightarrow 0} \sup _{x \in \Omega} \int_{|x-y|<\delta} \frac{|v(y)|}{|x-y|^{n-2}} d y=0,
$$

which B. Simon has conjectured is enough to guarantee unique continuation (cf. [Sa]). Part (A) of the following theorem improves the results of [JK] and $\left[\mathrm{St}_{1}\right]$ while part $(\mathrm{B})$ provides a global analogue. See also $[\mathrm{K}]$, where our proof of part (A) appears. Let $H^{2}\left(\mathbf{R}^{n}\right)$ (respectively $H_{\text {loc }}^{2}\left(\mathbf{R}^{n}\right)$ ) denote the Sobolev space of functions whose derivatives up to order two are in $L^{2}\left(\mathbf{R}^{n}\right)$ (respectively $\left.L_{\text {loc }}^{2}\left(\mathbf{R}^{n}\right)\right)$.

Theorem (1.2). Let $u \in H_{\text {loc }}^{2}\left(\mathbf{R}^{n}\right), n \geq 3$, satisfy $|\Delta u| \leq v|u|$.

(A) If $u$ vanishes to infinite order at some point $a \in \mathbf{R}^{n}$, i.e.,

$$
\lim _{R \rightarrow 0} R^{-m} \int_{|x-a|<R}|u(x)|^{2} d x=0 \text { for all } m>0,
$$

and if $v$ is locally small in $F_{p}$, i.e.,

$$
\varlimsup_{R \rightarrow 0}\left\|\chi_{\{x:|x-y|<R\}} v\right\|_{F_{p}} \leq \varepsilon(p, n) \quad \text { for all } y \in \mathbf{R}^{n},
$$

for some $p>(n-1) / 2$ and for a sufficiently small positive constant $\varepsilon(p, n)$ depending only on $p$ and $n$, then $u$ vanishes identically in $\mathbf{R}^{n}$.

(B) If $u \in H^{2}\left(\mathbf{R}^{n}\right)$ vanishes to infinite order at infinity, i.e.,

$$
\lim _{R \rightarrow \infty} R^{m} \int_{|x|>R}|u(x)|^{2} d x=0 \text { for all } m>0,
$$

and if $v$ satisfies

$$
\left\|\chi_{\{x:|x|>R\}} v\right\|_{F_{p}} \leq \varepsilon(p, n),
$$

for some $R>0$ and $p>(n-1) / 2$ and for a sufficiently small positive constant $\varepsilon(p, n)$ depending only on $p$ and $n$, then $u(x)$ vanishes identically for $|x|>R$.

Our next result is a variant of part (B) of Theorem (1.2) for the differential inequality (1.1) in which $u$ is permitted to merely vanish at infinity in a certain direction, provided it decays very rapidly in the opposite direction. To conserve notation we assume, without loss of generality, that $Q(D)=\Delta+a_{n} \partial / \partial x_{n}+\gamma$, $a_{n}$ real.

Theorem (1.3). Let $u \in H^{2}\left(\mathbf{R}^{n}\right), n \geq 3$, satisfy $|Q(D) u| \leq v|u|$ and suppose that

$$
\lim _{R \rightarrow \infty} \int_{R<|x|<2 R}|u(x)|^{2} e^{-\lambda x_{n}} d x=0 \text { for all } \lambda \text { sufficiently large. }
$$

If $v \in F_{p}$ satisfies $\left\|\chi_{\left\{x: x_{n}<t\right\}} v\right\|_{F_{p}} \leq \varepsilon(p, n)$ for some real $t$, where $\varepsilon(p, n)$ is a sufficiently small positive constant depending only on $p$ and $n$, then $u(x)$ vanishes identically for $x_{n}<t$. 
This theorem is a consequence of a weighted restriction theorem for the Fourier transform, Corollary (2.8), which includes the well-known result of E. Stein and P. Thomas (Corollary (2.9) below).

Finally we mention some further results for dimensions $n=2$ and 3. For $v \geq 0$ on $\mathbf{R}^{n}$, let $\|\mid v\| \|$ denote the least constant $C$ for which the following inequality holds:

$$
\left(\int_{\mathbf{R}^{n}}|f(x)|^{2} v(x) d x\right)^{1 / 2} \leq C\left(\int_{\mathbf{R}^{n}}|\nabla f|^{2}\right)^{1 / 2},
$$

for all $f \in C_{c}^{\infty}\left(\mathbf{R}^{n}\right)$. Equivalently $\|v\| \|$ is the norm of the embedding $I_{1}$ : $L^{2} \rightarrow L^{2}(v)$, where $I_{\alpha}$ denotes the fractional integral of order $\alpha$,

$$
I_{\alpha} f(x)=c_{\alpha, n} \int_{\mathbf{R}^{n}}|x-y|^{\alpha-n} f(y) d y, \quad 0<\alpha<n .
$$

The following theorem improves on all strong unique continuation results for the inequality $|\Delta u| \leq v|u|$ known in dimensions 2 and 3 , including Theorem (1.2)(A) and the results of [JK, Sa, and $\left.\mathrm{St}_{1}\right]$. Denote by $B(y, R)$ the ball of radius $R$ and centre $y$.

Theorem (1.4). Suppose $\Omega$ is a bounded, open and connected subset of $\mathbf{R}^{n}$, $n=2$ or 3 . Let $u \in H_{\mathrm{loc}}^{2}(\Omega)$ vanish to infinite order at some point in $\Omega$, and suppose that $|\Delta u| \leq v|u|$ in $\Omega$, where $v$ satisfies

$$
\varlimsup_{R \rightarrow 0}\left|\left\|\chi_{B(y, R)} v \mid\right\| \leq \varepsilon \quad \text { for all } y \in \Omega,\right.
$$

for a sufficiently small positive constant $\varepsilon$. Then $u$ vanishes identically in $\Omega$.

In [KS], it is shown that

$$
\||v|\| \approx \sup _{\text {cubes } Q} \frac{1}{\int_{Q} v(x) d x} \int_{Q} \int_{Q}|x-y|^{2-n} v(x) v(y) d x d y
$$

(with $|x-y|^{2-n}$ replaced by $\log |x-y|$ if $n=2$ ) and it is now clear that the Kato condition implies that of Theorem (1.4). In [F], it is shown that $\|v\|_{F_{1}} \leq\|v\|\left\|\leq c_{p}\right\| v \|_{F_{p}}, p>1$. It is an open question whether Theorem (1.4) remains valid for $n \geq 4$.

In dimension $n=2$, we prove a unique continuation theorem involving a gradient term.

Theorem (1.5). Suppose $\Omega$ is a bounded, open, and connected subset of $\mathbf{R}^{2}$. Let $u \in H_{\mathrm{loc}}^{2}(\Omega)$ vanish to infinite order at some point in $\Omega$, and suppose that $|\Delta u| \leq w|\nabla u|$ in $\Omega$, where $w^{2}$ is locally small in $F_{p / 2}$, i.e.,

$$
\varlimsup_{R \rightarrow 0} \sup _{\text {cubes } Q}|Q|\left(\frac{1}{|Q|} \int_{Q \cap\{x:|x-y|<R\}} w(x)^{p} d x\right)^{2 / p} \leq \varepsilon(p)
$$


for all $y \in \Omega$, for some $p>1$ and for a sufficiently small positive constant $\varepsilon(p)$ depending only on $p$. Then $u$ vanishes identically in $\Omega$.

The differential inequality $|\Delta u| \leq w|\nabla u|+v|u|$ is treated for $n \geq 3$ in [BKRS], where it is shown that uniqueness holds if $w \in L_{\mathrm{loc}}^{r}$ and $v \in L_{\mathrm{loc}}^{s}$, $r=(3 n-2) / 2, s>n / 2$. By Theorem (1.5), strong uniqueness holds for $|\nabla u| \leq w|\nabla u|$ if $w \in L_{\text {loc }}^{2}, n=2$. It is an open question whether uniqueness holds for $|\Delta u| \leq w|\nabla u|$ if $w \in L_{\text {loc }}^{n}, n \geq 3$. The proofs of Theorems (1.2) and (1.3) are in $\S 3$ and $\S 2$ respectively and the proofs of Theorems (1.4) and (1.5) are in $\S 4$.

We wish to thank S. Baouendi and Carlos Kenig for helpful conversations and for pointing out to us that some similar work has been done in [ChR].

We close this section by recording a pair of elementary inequalities that will be used in the next two sections.

Lemma (1.6). Let $f$ be in $H_{\mathrm{loc}}^{2}\left(\mathbf{R}^{n}\right), a>0$ and $\lambda \in \mathbf{R}$. Then

$$
\begin{aligned}
\int_{|x| \leq a}|\nabla f(x)|^{2} d x \leq & C\left(\int_{|x| \leq 2 a}|f(x)|^{2} d x\right)^{1 / 2}\left(\int_{|x| \leq 2 a}|\Delta f(x)|^{2} d x\right)^{1 / 2} \\
+ & C a^{-2} \int_{|x| \leq 2 a}|f(x)|^{2} d x \\
\int_{a \leq|x| \leq 2 a}|\nabla f(x)|^{2} e^{-\lambda x_{n}} d x \leq & C\left(\int_{a / 2 \leq|x| \leq 4 a}|f(x)|^{2} e^{-2 \lambda x_{n}} d x\right)^{1 / 2} \\
& \times\left(\int_{a / 2 \leq|x| \leq 4 a}|\Delta f(x)|^{2} d x\right)^{1 / 2} \\
& +C\left(a^{-2}+\lambda^{2}\right) \int_{a / 2 \leq|x| \leq 4 a}|f(x)|^{2} e^{-\lambda x_{n}} d x
\end{aligned}
$$

The constant $C$ is independent of $f, a$, and $\lambda$.

Proof. (A) It suffices to consider the case $a=1$. Let $\theta \in C_{c}^{\infty}(B(0,2))$ satisfy $\theta=1$ on $B(0,1)$. Then

$$
\int|\theta \nabla f|^{2} \leq 2 \int|f \nabla \theta|^{2}+2 \int|\nabla(\theta f)|^{2}=2 \int|f \nabla \theta|^{2}-2 \int \theta f \Delta(\theta f) .
$$

Now use

to get

$$
\begin{aligned}
|\theta f \Delta(\theta f)| & \leq|\theta f||\theta \Delta f|+2|f \nabla \theta\|\theta \nabla f|+\theta| \Delta \theta\| f|^{2} \\
& \leq|\theta f||\theta \Delta f|+16|\nabla \theta|^{2}|f|^{2}+\frac{1}{4}|\theta \nabla f|^{2}+\theta|\Delta \theta||f|^{2}
\end{aligned}
$$

$$
\begin{aligned}
\int|\theta \nabla f|^{2} \leq C & {\left[\int\left(|\nabla \theta|^{2}+\theta|\Delta \theta|\right)|f|^{2}+\left(\int|\theta f|^{2}\right)^{1 / 2}\left(\int|\theta \Delta f|^{2}\right)^{1 / 2}\right] } \\
& +\frac{1}{2} \int|\theta \nabla f|^{2}
\end{aligned}
$$

and then subtract the last summand from both sides. 
(B) Take $a=1$ and let $\varphi \in C_{c}^{\infty}\left(B(0,4) \backslash B\left(0, \frac{1}{2}\right)\right)$ satisfy $\varphi=1$ on $B(0,2) \backslash B(0,1)$. Set $\theta=e^{-(\lambda / 2) x_{n}} \varphi$. Arguing as in the proof of part (A) yields the following variant of (1.7),

$$
\begin{aligned}
\int|\theta \nabla f|^{2} \leq C & {\left[\int\left(|\nabla \theta|^{2}+\theta|\Delta \theta|\right)|f|^{2}+\left(\int\left|\theta^{2} f\right|^{2}\right)^{1 / 2}\left(\int_{1 / 2 \leq|x| \leq 4}|\Delta f|^{2}\right)^{1 / 2}\right] } \\
+ & \frac{1}{2} \int|\theta \nabla f|^{2} .
\end{aligned}
$$

Now use $|\theta| \leq C e^{-(\lambda / 2) x_{n}}, \quad|\nabla \theta| \leq C(1+|\lambda|) e^{-(\lambda / 2) x_{n}}$ and $|\Delta \theta| \leq$ $C\left(1+\lambda^{2}\right) e^{-(\lambda / 2) x_{n}}$.

\section{Proof of Theorem (1.3)}

We follow the argument of [KRS] beginning with a weighted $L^{2}$ version of their Carleman inequality.

Lemma (2.1). Let $Q(D)=\Delta+a_{n} \partial / \partial x_{n}+\gamma$ where $n \geq 3, a_{n} \in \mathbf{R}$ and $\gamma \in \mathbf{C}$. Suppose $v \geq 0$ is in $F_{p}$ with $p>(n-1) / 2$. Then for $f \in C_{0}^{\infty}\left(\mathbf{R}^{n}\right)$ and $\lambda$ real,

$$
\int_{\mathbf{R}^{n}}|f|^{2} e^{-2 \lambda x_{n}} v d x \leq C \int_{\mathbf{R}^{n}}|Q(D) f|^{2} e^{-2 \lambda x_{n}} v^{-1} d x
$$

with $C$ independent of $f, \lambda, a_{n}$, and $\gamma$.

We begin with a weighted restriction theorem which will substitute for Lemma 2.1(a) of [KRS]. Let $J_{\beta}$ denote the Bessel function of order $\beta$.

Lemma (2.2). Let $n \geq 2$ and $2 n /(n+1) \leq \delta \leq(n+1) / 2$. Set $p_{0}=$ $(n-1) / 2(\delta-1)$ and let $p>p_{0}$ if $\delta>2 n /(n+1)$ and $p=p_{0}$ if $\delta=$ $2 n /(n+1)$. Suppose $v \geq 0$ and that for all cubes $Q \subset \mathbf{R}^{n}$,

$$
|Q|^{\delta / n}\left(\frac{1}{|Q|} \int_{Q} v^{p} d x\right)^{1 / p} \leq C
$$

Then

$$
\int_{\mathbf{R}^{n}}|f * \widehat{d \sigma}|^{2} v d x \leq C \int_{\mathbf{R}^{n}}|f|^{2} v^{-1} d x
$$

where

$$
\widehat{d \sigma}(x)=\int_{S^{n-1}} e^{i\langle x, \xi\rangle} d \sigma(\xi)=c_{n} J_{(n-2) / 2}(|x|)|x|^{-(n-2) / 2} .
$$

Proof. Before proving the lemma we note that the restriction $\delta \leq(n+1) / 2$ is to ensure that $p_{0} \geq 1$. We consider first the case $\delta>2 n /(n+1)$. Let $v^{*}(x)=M\left(v^{q}\right)^{1 / q}(x)$, where $p>q>p_{0}$ and where $M$ is the Hardy-Littlewood maximal function. Define

$$
T_{z} f(x)=\left[K_{z} *\left(f v^{* z / 2}\right)(x)\right] v^{*}(x)^{z / 2}, \quad \text { where } K_{z}(x)=\frac{J_{n / 2-z}(|x|)}{|x|^{n / 2-z}} .
$$


We will apply Stein's theorem on complex interpolation of operators [ $\left[\mathrm{St}_{2}\right]$. First, for $\operatorname{Re} z=0$, we have by [T],

$$
\int_{\mathbf{R}^{n}}\left|T_{z} f\right|^{2} d x \leq C_{z} \int_{\mathbf{R}^{n}}|f|^{2} d x .
$$

Let $\operatorname{Re} z=p_{0}$ and $\alpha=(n-1) / 2+p_{0}$. Since $\left|K_{z}(x)\right| \leq C_{z}|x|^{-(n+1) / 2+p_{0}}$, we have $\alpha<n$ and

$$
\left|T_{z} f(x)\right| \leq C_{z} I_{\alpha}\left(|f| v^{* p_{0} / 2}\right) v^{* p_{0} / 2}(x) .
$$

We have used the estimate $\left|J_{\beta}(|x|)\right| \leq C_{\beta}|x|^{-1 / 2}$ for $\operatorname{Re} \beta \geq-1 / 2$, which applies here as $p_{0} \leq(n+1) / 2$ for $\delta \geq 2 n /(n+1)$. Thus, for $\operatorname{Re} z=p_{0}$, $\alpha=(n-1) / 2+p_{0}$, from (2.4) we get,

$$
\int_{\mathbf{R}^{n}}\left|T_{z} f\right|^{2} d x \leq C_{z} \int_{\mathbf{R}^{n}}\left[I_{\alpha}\left(|f| v^{* p_{0} / 2}\right)\right]^{2} v^{* p_{0}} d x .
$$

By [CR], $v^{* p_{0}} \in A_{1}$, and $v^{*-p_{0}} \in A_{2}$ and so by [CW],

$$
\int_{\mathbf{R}^{n}}\left[I_{\alpha}\left(|f| v^{* p_{0} / 2}\right)\right]^{2} v^{* p_{0}} \leq C \int_{\mathbf{R}^{n}}\left|f v^{* p_{0} / 2}\right|^{2}\left(v^{*}\right)^{-p_{0}}=C \int_{\mathbf{R}^{n}}|f|^{2} d x
$$

provided, for $r>1$,

$$
|Q|^{2 \alpha / n}\left(\frac{1}{|Q|} \int_{Q} v^{* r p_{0}}\right)^{1 / r} \leq \frac{1}{|Q|} \int_{Q} v^{*-p_{0}} .
$$

Because $v^{*-p_{0}} \in A_{2},(2.6)$ is equivalent to

$$
|Q|^{2 \alpha / n}\left(\frac{1}{|Q|} \int_{Q} v^{* r p_{0}}\right)^{2 / r} \leq C .
$$

Taking square roots and setting $r p_{0}=p$ and noting $\alpha / p_{0}=\delta$, we get that (2.5) follows, provided

$$
|Q|^{\alpha / n}\left(\frac{1}{|Q|} \int_{Q} v^{* p}\right)^{1 / p} \leq C, \quad p_{0}<q<p
$$

To see (2.7), we use the argument of [F, proof of Lemma C]. Set $v_{1}=v \chi_{2 Q}$, $v_{2}=v-v_{1}$. Clearly, the left side of (2.7) is bounded by

$$
|Q|^{\delta / n}\left(\frac{1}{|Q|} \int_{Q} v_{1}^{* p}\right)^{1 / p}+|Q|^{\delta / n}\left(\frac{1}{|Q|} \int_{Q} v_{2}^{* p}\right)^{1 / p} .
$$

By the definition of $v_{2}$, for $x \in Q$,

$$
v_{2}^{*}(x) \leq\left(\frac{1}{|B|} \int_{B}\left|v_{2}\right|^{q}\right)^{1 / q}
$$

for some cube $B \supset Q$. Thus because $p>q$,

$$
|Q|^{\delta / n}\left(\frac{1}{|Q|} \int_{Q} v_{2}^{* p}\right)^{1 / p} \leq|B|^{\delta / n}\left(\frac{1}{|B|} \int_{B} v_{2}^{q}\right)^{1 / q} \leq|B|^{\delta / n}\left(\frac{1}{|B|} \int_{B} v^{q}\right)^{1 / q} \leq C .
$$


By the maximal theorem,

$$
\begin{aligned}
|Q|^{\delta / n}\left(\frac{1}{|Q|} \int_{Q} v_{1}^{* p}\right)^{1 / p} & \leq|Q|^{\delta / n}\left(\frac{1}{|Q|} \int_{\mathbf{R}^{n}} v_{1}^{* p}\right)^{1 / p} \\
& \leq|Q|^{\delta / n}\left(\frac{1}{|Q|} \int_{\mathbf{R}^{n}} v_{1}^{p}\right)^{1 / p} \\
& \leq|Q|^{\delta / n}\left(\frac{1}{|Q|} \int_{2 Q} v^{p}\right)^{1 / p} \leq C .
\end{aligned}
$$

Thus (2.7) is proved. Therefore (2.3) holds for $\operatorname{Re} z=p_{0}$, at least in the case $\delta>2 n /(n+1)$. In the case $\delta=2 n /(n+1), p=p_{0}$, set

$$
T_{z} f(x)=\left[K_{z} *\left(f v^{z / 2}\right)(x)\right] v^{z / 2}(x) .
$$

Inequality (2.3) persists for $\operatorname{Re} z=0$. Since $\left|K_{z}(x)\right| \leq C_{z}$ for $\operatorname{Re} z=p_{0}=$ $(n+1) / 2$,

$$
\left|T_{z} f(x)\right|^{2} \leq\left[\int|f|^{2} d x\right]\left[\int v^{(n+1) / 2} d x\right] v(x)^{(n+1) / 2}
$$

by Hölder's inequality and so $(2.3)$ holds for $\operatorname{Re} z=p_{0}=(n+1) / 2$ if

$$
\int v^{(n+1) / 2} d x<\infty
$$

as required. By interpolation, (2.3) holds for $\operatorname{Re} z=1$. Thus

$$
\begin{aligned}
\int_{\mathbf{R}^{n}}|f * \widehat{d \sigma}|^{2} v & \leq \int_{\mathbf{R}^{n}}|f * \widehat{d \sigma}|^{2} v^{*}=\int_{\mathbf{R}^{n}}\left|T_{1}\left(f v^{*-1 / 2}\right)\right|^{2} d x \\
& \leq C \int_{\mathbf{R}^{n}}|f|^{2} v^{*-1} d x \leq C \int_{\mathbf{R}^{n}}|f|^{2} v^{-1} d x
\end{aligned}
$$

and the lemma is proved.

We now obtain the corollaries that follow.

Corollary (2.8). Let $v$ satisfy the hypothesis of Lemma (2.2). Then,

$$
\int_{S^{n-1}}|\hat{f}|^{2} d \sigma \leq C \int_{\mathbf{R}^{n}}|f|^{2} v^{-1} d x .
$$

Proof. We use P. Tomas's duality argument.

$$
\int_{S^{n-1}}|\hat{f}|^{2} d \sigma=\int_{\mathbf{R}^{n}} f(\bar{f} * \widehat{d \sigma}) \leq\|f\|_{L_{v^{-1}}^{2}}\|f * \widehat{d \sigma}\|_{L_{v}^{2}} .
$$

By Lemma (2.2),

$$
\|f * \widehat{d \sigma}\|_{L_{v}^{2}} \leq C\|f\|_{L_{v-1}^{2}},
$$

and the corollary now follows.

Corollary (2.9) [T]. Let $n \geq 2$ and $1 \leq p \leq 2(n+1) /(n+3)$. Then

$$
\left(\int_{S^{n-1}}|\hat{f}|^{2} d \sigma\right)^{1 / 2} \leq C_{p}\left(\int_{\mathbf{R}^{n}}|f|^{p}\right)^{1 / p} .
$$


Proof. Set $v(x)=|f|^{2-p}$ and assume $\|f\|_{p}=1$. Note that

$$
\int_{\mathbf{R}^{n}}|f|^{p} d x=\int_{\mathbf{R}^{n}}|f|^{2} v^{-1} d x
$$

If $v \in L^{n / \delta}$, then the hypothesis of Corollary (2.8) will be fulfilled and we would have

$$
\int_{S^{n-1}}|\hat{f}|^{2} d \sigma \leq C \int_{\mathbf{R}^{n}}|f|^{2} v^{-1}=C \int_{\mathbf{R}^{n}}|f|^{p}=C .
$$

For $v \in L^{n / \delta}\left(\mathbf{R}^{n}\right)$, we need $|f|^{(2-p) n / \delta}=|f|^{p}$, or $(2-p) n / \delta=p$. This forces $p=2 n /(n+\delta)$. But for $\delta \geq 2 n /(n+1), p \leq 2(n+1) /(n+3)$ and we are done.

The result of Corollary (2.9) was obtained earlier by E. Stein and P. Tomas [T]. We remark that the sharp result with $p=2(n+1) /(n+3)$ in Corollary (2.9) is equivalent to the case $\delta=2 n /(n+1)$ of Lemma (2.2) since Holder's inequality shows that

$$
\left[\int|f|^{p} d x\right]^{1 / p} \leq\left[\int|f|^{2} v^{-1} d x\right]^{1 / 2}\left[\int v^{(n+1) / 2} d x\right]^{1 /(n+1)}
$$

if $p=2(n+1) /(n+3)$. However, we shall be interested in the special case $\delta=2$ of Lemma (2.2), which neither implies nor is implied by Corollary (2.9). Our next result serves as a substitute for Lemma 2.1(b) of [KRS].

Lemma (2.10). Let $v \in F_{p}, p>(n-1) / 2$. Suppose $\operatorname{Im} z \neq 0$ and $\operatorname{Re} z<0$ and let

$$
\widehat{T f}(\xi)=\frac{\hat{f}(\xi)}{|\xi|^{2}+z} .
$$

Then,

$$
\int_{\mathbf{R}^{n}}|T f|^{2} v d x \leq C \int_{\mathbf{R}^{n}}|f|^{2} v^{-1} d x
$$

with $C$ independent of $z$.

Proof. We mimic the proof of Lemma (2.2) in the special case $\delta=2$. Let $v^{*}(x)$ be as in Lemma (2.2) with $p_{0}=(n-1) / 2$. Clearly it is enough to show

$$
\int_{\mathbf{R}^{n}}|T f|^{2} v^{*} d x \leq c \int_{\mathbf{R}^{n}}|f|^{2} v^{*-1} d x .
$$

Define

$$
\widehat{T_{\mu}} f(\xi)=\frac{\hat{f}(\xi)}{\left(|\xi|^{2}+z\right)^{\mu}}, \quad \operatorname{Im} z \neq 0, \operatorname{Re} z<0,
$$

and let $G_{\mu} f(x)=\left[T_{\mu}\left(f v^{* \mu / 2}\right)\right] v^{* \mu / 2}(x)$.

Note that if $\operatorname{Re} \mu=0$ and $\operatorname{Im} z \neq 0$ we have $\left\|G_{\mu} f\right\|_{2} \leq\|f\|_{2}$. If $\operatorname{Re} \mu=$ $(n-1) / 2$, the kernel $R_{\mu}(x)$ of $T_{\mu}$ satisfies

$$
\left|R_{\mu}(x)\right| \leq C|x|^{-1} .
$$


This is seen by examining the formula for $R_{\mu}(x)$ in [KRS and GS]. In fact,

$$
R_{\mu}(x)=\frac{e^{\mu^{2}} 2^{-\mu+1}}{(2 \pi)^{n / 2} \Gamma(\mu) \Gamma(n / 2-\mu)}\left[\frac{z}{|x|^{2}}\right]^{(1 / 2)(n / 2-\mu)} K_{n / 2-\mu}\left(\sqrt{z|x|^{2}}\right)
$$

where $K_{\delta}$ is the Bessel kernel of the third kind. It is known that

$$
\left|K_{\delta}(w)\right| \leq \begin{cases}c|w|^{-|\operatorname{Re} \delta|}, & \text { if }|w| \leq 1, \operatorname{Re} w>0 \\ c|w|^{-1 / 2}, & \text { if }|w| \geq 1, \operatorname{Re} w>0 .\end{cases}
$$

If $\delta=n / 2-\mu, \operatorname{Re} \mu=(n-1) / 2$ and $w=\sqrt{z|x|^{2}}$, we see $\operatorname{Re} \delta=1 / 2$ and $\operatorname{Re} w>0$ as $\operatorname{Re} z<0$. Therefore we may estimate (2.12) by (2.13) to get (2.11). Thus if $\operatorname{Re} \mu=(n-1) / 2$,

$$
\left|G_{\mu} f(x)\right| \leq I_{n-1}\left(|f| v^{*(n-1) / 4}\right) v^{*(n-1) / 4},
$$

and so

$$
\int_{\mathbf{R}^{n}}\left|G_{\mu} f\right|^{2} d x \leq \int_{\mathbf{R}^{n}}\left[I_{n-1}\left(|f| v^{*(n-1) / 4}\right)\right]^{2} v^{*(n-1) / 2} d x .
$$

But by the arguments of Lemma (2.2), the integral on the right side above is at most $\int_{\mathbf{R}^{n}}|f|^{2} d x$ because $v^{*}=\left(M v^{q}\right)^{1 / q} \in F_{p}$ for $1<q<p$ (see the argument following (2.7) or the proof of Lemma C, p. 153, in [F]). The lemma now follows using complex interpolation, and then taking $\mu=1$.

The next lemma is needed to adapt the Littlewood-Paley arguments of [KRS] to the weighted setting. Note the restriction to $p>(n-1) / 2$.

Lemma (2.14). Let $v \in F_{p}, p>(n-1) / 2$. For $s \geq 1$, define the onedimensional maximal function $v_{*}(x)$ by

$$
v_{*}(x)=\sup _{\mu}\left(\frac{1}{2 \mu} \int_{x_{n}-\mu}^{x_{n}+\mu} v\left(x_{1}, x_{2}, \ldots, x_{n-1}, t\right)^{s} d t\right)^{1 / s} .
$$

Then, for $p>\max (s,(n-1) / 2)$

$$
\sup _{Q}|Q|^{2 / n}\left(\frac{1}{|Q|} \int_{Q} v_{*}^{p}\right)^{1 / p} \leq C \sup _{Q}|Q|^{2 / n}\left(\frac{1}{|Q|} \int_{Q} v^{p}\right)^{1 / p} .
$$

That is, $v_{*} \in F_{p}$.

Proof. Fix a cube $Q$ with center at $z$ and edge length $\delta$. Define the rectangle $R_{k}, k \geq 1$, such that $\left(y_{1}, y_{2}, \ldots, y_{n-1}, y_{n}\right) \in R_{k}$ if $\left|y_{j}-z_{j}\right| \leq 2 \delta, j=$ $1,2, \ldots, n-1$, but $2^{k} \delta<\left|y_{n}-z_{n}\right| \leq 2^{k+1} \delta$. Let $R_{0}=4 Q$. Let $v^{(k)}=v \chi_{R_{k}}$. Then, $v(x) \leq \sum_{k=0}^{\infty} v^{(k)}(x)+\varphi(x)$, where $\varphi(x)$ is supported on $\mathbf{R}^{n} \backslash \bigcup_{k=0}^{\infty} R_{k}$. Thus,

$$
|Q|^{2 / n}\left(\frac{1}{|Q|} \int_{Q} v_{*}^{p}\right)^{1 / p} \leq \sum_{k=0}^{\infty}|Q|^{2 / n}\left(\left(\frac{1}{|Q|} \int_{Q} v_{*}^{(k) p}\right)^{1 / p}+\left(\frac{1}{|Q|} \int_{Q} \varphi_{*}^{p}\right)^{1 / p}\right) .
$$


But for $x \in Q, \varphi_{*}(x)=0$. By the maximal theorem when $k=0$,

$$
|Q|^{2 / n}\left(\frac{1}{|Q|} \int_{Q} v_{*}^{(0) p}\right)^{1 / p} \leq C|Q|^{2 / n}\left(\frac{1}{|Q|} \int_{4 Q} v^{p}\right)^{1 / p} \leq c .
$$

If $k \neq 0$, note that for $\left(x_{1}, \ldots, x_{n}\right) \in Q$,

$$
v_{*}^{(k)}(x)^{p} \leq \frac{C}{2^{k} \delta} \int_{2^{k} \delta<\left|z_{n}-t\right| \leq 2^{k+1} \delta} v\left(x_{1}, \ldots, x_{n-1}, t\right)^{p} d t .
$$

Thus for $k \neq 0$

$$
\frac{1}{|Q|} \int_{Q} v_{*}^{(k) p} d x \leq \frac{C}{2^{k} \delta^{n}} \int_{R_{k}} v^{p} d x .
$$

Now there exists a cube $Q_{k}$ such that $Q_{k} \supset R_{k}$ and $\left|Q_{k}\right| \sim\left(2^{k} \delta\right)^{n}$. Thus

$$
\frac{c}{\delta^{n-1}\left(2^{k} \delta\right)} \int_{R^{k}} v^{p} d x \leq C 2^{(n-1) k} \frac{1}{\left|Q_{k}\right|} \int_{Q_{k}} v^{p} d x .
$$

Moreover $|Q|^{2 / n}=2^{-2 k}\left|Q_{k}\right|^{2 / n}$. Using this and the fact that for all $k$

$$
\left(\frac{1}{|Q|} \int_{Q} v_{*}^{(k) p}\right)^{1 / p} \leq C 2^{(n-1) k / p}\left(\frac{1}{\left|Q_{k}\right|} \int_{Q_{k}} v^{p} d x\right)^{1 / p},
$$

the right side of $(2.15)$ is at most $C \sum_{k=0}^{\infty} 2^{(-2+(n-1) / p) k}$. This converges provided $p>(n-1) / 2$.

Proof of Lemma (2.1). We follow the argument of [KRS]. Conjugating the operator $Q(D)$ by $e^{-\lambda x_{n}}$ we see that, if $\gamma=\alpha+i \mu$, we have

$$
\begin{aligned}
\left(e^{-\lambda x_{n}} Q(D) e^{\lambda x_{n}}\right) \hat{\top}(\xi) & =-\left(|\xi|^{2}-\lambda^{2}-a_{n} \lambda-i \xi_{n}\left(2 \lambda-a_{n}\right)-\gamma\right) \\
& =-\left(|\xi|^{2}-\lambda^{2}-a_{n} \lambda-\alpha-2 i \lambda\left(\xi_{n}-\frac{a_{n} \lambda^{-1}}{2}+\frac{\mu \lambda^{-1}}{2}\right)\right) \\
& =-\left(|\xi|^{2}-\lambda^{2}-a_{n} \lambda-\alpha-2 i \lambda\left(\xi_{n}+\delta\right)\right),
\end{aligned}
$$

where $\delta=-a_{n} \lambda^{-1} / 2+\mu \lambda^{-1} / 2$. Thus it will be enough to show,

$$
\int_{\mathbf{R}^{n}}|S f|^{2} v d x \leq c \int_{\mathbf{R}^{n}}|f|^{2} v^{-1} d x
$$

where

$$
\widehat{S f}(\xi)=\hat{f}(\xi)\left[|\xi|^{2}-\lambda^{2}-a_{n} \lambda-\alpha-2 i \lambda\left(\xi_{n}+\delta\right)\right]^{-1}=\hat{f}(\xi) m(\xi) .
$$

Let $\chi_{k}\left(\xi_{n}\right)=\chi\left(2^{-k}\left(\xi_{n}+\delta\right)\right)$ be a smooth Littlewood-Paley-Stein decomposition of $\mathbf{R}$. Let $\widehat{R_{k}} f(\xi)=\chi_{k}\left(\xi_{n}\right) m(\xi) \hat{f}(\xi)$. Define $v_{*}$ as in Lemma (2.14) for some $s$ with $1<s<p$. Then $v_{*}(x) \in A_{1}\left(\mathbf{R}, d x_{n}\right)$. Thus by applying [Ku] to the $x_{n}$ variable,

$$
\int_{\mathbf{R}^{n}}|S f|^{2} v d x \leq \int_{\mathbf{R}^{n}}|S f|^{2} v_{*} d x \leq \sum_{k} \int_{\mathbf{R}^{n}}\left|R_{k} f\right|^{2} v_{*} d x
$$


Let $\hat{f}_{k}(\xi)=\chi_{k}\left(\xi_{n}\right) \hat{f}(\xi)$. We claim

$$
\int_{\mathbf{R}^{n}}\left|R_{k} f\right|^{2} v_{*} d x \leq C \int_{\mathbf{R}^{n}}\left|f_{k}\right|^{2} v_{*}^{-1} d x
$$

with a constant $c$ independent of $k$ and $\lambda$. Now $v_{*}^{-1} \in A_{2}\left(\mathbf{R}, d x_{n}\right)$. Assuming (2.18), the weighted Littlewood-Paley-Stein theorem of $[\mathrm{Ku}]$ shows that

$$
\begin{aligned}
\sum_{k} \int_{\mathbf{R}^{n}}\left|R_{k} f\right|^{2} v_{*} d x & \leq C \sum_{k} \int_{\mathbf{R}^{n}}\left|f_{k}\right|^{2} v_{*}^{-1} d x \\
& \leq C \int_{\mathbf{R}^{n}}|f|^{2} v_{*}^{-1} d x \leq c \int_{\mathbf{R}^{n}}|f|^{2} v^{-1} d x .
\end{aligned}
$$

Combining this with (2.17) yields $(2.16)$.

We now prove (2.18). We have $R_{k}=A_{k}+B_{k}$ with

$$
\begin{gathered}
\widehat{A_{k} f}(\xi)=\frac{\chi_{k}\left(\xi_{n}\right)}{|\xi|^{2}-\lambda^{2}-a_{n} \lambda-\alpha-i 2^{k+1} \lambda} \hat{f}(\xi), \\
\widehat{B_{k} f}(\xi)=\chi_{k}\left(\xi_{n}\right)\left[\frac{1}{|\xi|^{2}-\lambda^{2}-\alpha_{n} \lambda-\alpha-2 i \lambda\left(\xi_{n}+\delta\right)}\right. \\
\left.-\frac{1}{|\xi|^{2}-\lambda^{2}-a_{n} \lambda-\alpha-i 2^{k+1} \lambda}\right] \hat{f}(\xi) .
\end{gathered}
$$

Observe that

$$
\widehat{A_{k} f}(\xi)=\frac{1}{|\xi|^{2}+z} \chi_{k}\left(\xi_{n}\right) \hat{f}(\xi)
$$

with $\operatorname{Re} z<0, \operatorname{Im} z \neq 0$.

By Lemma (2.14), $v_{*} \in F_{p}, p>(n-1) / 2$. If $T$ is as in Lemma (2.10), we have

$$
\int_{\mathbf{R}^{n}}\left|A_{k} f\right|^{2} v_{*}=c \int_{\mathbf{R}^{n}}\left|T f_{k}\right|^{2} v_{*} \leq c \int_{\mathbf{R}^{n}}\left|f_{k}\right|^{2} v_{*}^{-1} .
$$

As in [KRS], we write $B_{k} f(x)$ in polar coordinates with $|\xi|=r$,

$$
\begin{aligned}
B_{k} f(x)=\int_{0}^{\infty} \int_{S^{n-1}} e^{i\langle r \sigma, x\rangle} \varphi_{2}(r) \chi_{k}\left(r \sigma_{n}\right) \hat{f}(r \sigma) & \\
\times & {\left[\frac{1}{r^{2}-\lambda^{2}-a_{n} \lambda-\alpha-2 i \lambda\left(r \sigma_{n}+\delta\right)}\right.} \\
& \left.-\frac{1}{r^{2}-\lambda^{2}-a_{n} \lambda-\alpha-i 2^{k+1} \lambda}\right] r^{n-1} d \sigma d r .
\end{aligned}
$$

Thus,

with

$$
\left|B_{k} f(x)\right| \leq 2 \int_{0}^{\infty}\left|\int_{S^{n-1}} m_{k, r}(r \sigma) \hat{f}_{k}(r \sigma) e^{i\langle\sigma, r x\rangle} d \sigma\right| r^{n-1} d r
$$

$$
m_{k, r}(\xi)=\frac{\lambda\left(\xi_{n}+\delta-2^{k}\right) \psi_{k}\left(\xi_{n}\right)}{\left(r^{2}-\lambda^{2}-a_{n} \lambda-\alpha-2 i \lambda\left(\xi_{n}+\delta\right)\right)\left(r^{2}-\lambda^{2}-a_{n} \lambda-\alpha-i 2^{k+1} \lambda\right)},
$$


where $\psi_{k} \equiv 1$ on $\operatorname{supp} \chi_{k}$ and $\psi_{k}$ supported near $\left|\xi_{n}+\delta\right| \approx 2^{k}$. Thus,

$$
\left|B_{k} f(x)\right| \leq C \int_{0}^{\infty}\left|\delta_{r}\left(T_{m_{k}, r} f_{k}\right) * \widehat{d \sigma}(r x)\right| r^{n-1} d r,
$$

where $\delta_{r} h(x)=\left(1 / r^{n}\right) h(x / r)$ and $T_{m_{k, r}}$ denotes the multiplier with symbol $m_{k, r}$. By Minkowski's inequality,

$$
\left(\int_{\mathbf{R}^{n}}\left|B_{k} f\right|^{2} v * d x\right)^{1 / 2} \leq C \int_{0}^{\infty}\left(\int_{\mathbf{R}^{n}}\left|\delta_{r}\left(T_{m_{k, r}} f_{k}\right) * \widehat{d \sigma}(r x)\right|^{2} v_{*}(x)\right)^{1 / 2} r^{n-1} d r .
$$

Changing variables by setting $r x=y$, the last integral is

$$
C \int_{0}^{\infty}\left(\int_{\mathbf{R}^{n}}\left|\delta_{r}\left(T_{m_{k, r}} f_{k}\right) * \widehat{d \sigma}(y)\right|^{2} v_{*}\left(\frac{y}{r}\right) d y\right)^{1 / 2} r^{n / 2-1} d r .
$$

Let $w(y)=\left(1 / r^{2}\right) v_{*}(y / r)$. Then by Lemma (2.14) and a rescaling,

$$
\left(\frac{1}{|Q|} \int_{Q} w^{p}\right)^{1 / p}|Q|^{2 / n} \leq C .
$$

Thus by Lemma (2.2) the integral above is dominated by

$$
C \int_{0}^{\infty}\left(\int_{\mathbf{R}^{n}} r^{-2 n}\left|T_{m_{k, r}} f_{k}\left(\frac{x}{r}\right)\right|^{2} v_{*}^{-1}\left(\frac{x}{r}\right) d x\right)^{1 / 2} r^{n / 2+1} d r,
$$

and by changing variables again this integral is at most

$$
\int_{0}^{\infty}\left(\int_{\mathbf{R}^{n}}\left|T_{m_{k, r}} f_{k}(x)\right|^{2} v_{*}^{-1}(x) d x\right)^{1 / 2} r d r .
$$

Now, one easily checks that,

$$
\left|m_{k, r}\left(\xi_{n}\right)\right| \leq \frac{C \lambda 2^{k}}{\left(r^{2}-\lambda^{2}-a_{n} \lambda-\alpha\right)^{2}+2^{2 k} \lambda^{2}}
$$

and

$$
\left|m_{k, r}^{\prime}\left(\xi_{n}\right)\right| \leq \frac{C \lambda}{\left(r^{2}-\lambda^{2}-a_{n} \lambda-\alpha\right)^{2}+2^{2 k} \lambda^{2}} .
$$

Thus $m_{k, r}\left(\xi_{n}\right)$ satisfies the conditions of the Marcinkiewicz multiplier theorem. Since $v_{*}^{-1} \in A_{2}\left(\mathbf{R}, d x_{n}\right)$, by [Ku], (2.19) above is at most

$$
\begin{aligned}
& C\left(\int_{\mathbf{R}^{n}}\left|f_{k}\right|^{2} v_{*}^{-1} d x\right)^{1 / 2} \int_{0}^{\infty} \frac{\lambda 2^{k} r}{\left(r^{2}-\lambda^{2}-a_{n} \lambda-\alpha\right)^{2}+2^{2 k} \lambda^{2}} d r \\
& \quad=C\left(\int_{\mathbf{R}^{n}}\left|f_{k}\right|^{2} v_{*}^{-1} d x\right)^{1 / 2} \int_{-\infty}^{\infty} \frac{d t}{t^{2}+1}, \quad 2^{k} \lambda t=r^{2}-\lambda^{2}-a_{n} \lambda-\alpha, \\
& \quad=\pi C\left(\int_{\mathbf{R}^{n}}\left|f_{k}\right|^{2} v_{*}^{-1} d x\right)^{1 / 2} .
\end{aligned}
$$

This proves (2.18) and, as mentioned earlier, completes the proof of Lemma (2.1). 
Proof of Theorem (1.3). Since $|Q(D) u| \leq v|u| \leq\left(v+\varepsilon /|x|^{2}\right)|u|$, and $v+\varepsilon /|x|^{2}$ also satisfies the hypothesis of Theorem (1.2), we may assume $v(x) \geq \varepsilon|x|^{-2}$. Select $\eta \in C_{0}^{\infty}(B(0,2))$ with $\eta \equiv 1$ on $B(0,1)$, set $\eta_{R}(x)=\eta(x / R)$ and let $S_{t}=\left\{\left(x_{1}, \ldots, x_{n}\right), x_{n}<t\right\}$. By hypothesis, $\left\|\chi_{S_{t}} v\right\|_{F_{p}} \leq \varepsilon(p, n)$ and thus the constant $C$ in the conclusion of Lemma (2.1) may be taken to be at most $c_{0}<1$ if $v$ is replaced by $\chi_{S_{t}} v$. Thus with $\lambda>0$ in Lemma (2.1),

$$
\begin{aligned}
& \int_{S_{l}}\left|\eta_{R} u\right|^{2} e^{-\lambda x_{n}} v d x \leq c_{0} \int_{\mathbf{R}_{+}^{n}}\left|Q(D)\left(\eta_{R} u\right)\right|^{2} e^{-\lambda x_{n}} v^{-1} d x \\
& \quad \leq c_{0}\left\{\int_{\mathbf{R}^{n}}\left|\eta_{R} Q(D) u\right|^{2} e^{-\lambda x_{n}} v^{-1} d x+C \int_{R \leq|x| \leq 2 R}\left(|u|^{2}+|\nabla u|^{2}\right) e^{-\lambda x_{n}} d x\right\}
\end{aligned}
$$

since $v^{-1} \leq C|x|^{2},\left|\nabla \eta_{R}\right| \leq C R^{-1}$ and $\left|\Delta \eta_{R}\right| \leq C R^{-2}$. For the first term on the right side of (2.20), the differential inequality yields

$$
\int_{\mathbf{R}^{n}}\left|\eta_{R} Q(D) u\right|^{2} e^{-\lambda x_{n}} v^{-1} d x \leq \int_{\mathbf{R}^{n} \backslash S_{t}}\left|\eta_{R} u\right|^{2} e^{-\lambda x_{n}} v d x+\int_{S_{t}}\left|\eta_{R} u\right|^{2} e^{-\lambda x_{n}} v d x .
$$

Since $\chi_{S_{t}} v \in F_{p}, p>1$, we have by $[\mathrm{F}]$

$$
\int_{S_{t}}\left|\eta_{R} u\right|^{2} e^{-\lambda x_{n}} v \leq e^{2|\lambda| R} \int_{S_{t}}\left|\eta_{R} u\right|^{2} v \leq C e^{2|\lambda| R} \int_{\mathbf{R}^{n}}\left|\nabla\left(\eta_{R} u\right)\right|^{2},
$$

and thus the second integral on the right side of (2.21) is finite. So from (2.20) we conclude

$$
\begin{aligned}
\int_{S_{t}}\left|\eta_{R} u\right|^{2} e^{-\lambda x_{n}} v d x \leq & C \int_{\mathbf{R}^{n} \backslash S_{t}}\left|\eta_{R} u\right|^{2} e^{-\lambda x_{n}} v d x \\
& +C \int_{R \leq|x| \leq 2 R}\left(|u|^{2}+|\nabla u|^{2}\right) e^{-\lambda x_{n}} d x .
\end{aligned}
$$

Multiplying by $e^{\lambda t}$ and using $[\mathrm{F}]$ again, we have

$$
\begin{gathered}
\int_{S_{t} \cap B_{R}}|u|^{2} e^{\lambda\left(t-x_{n}\right)} v d x \leq C \int_{\mathbf{R}^{n}}\left|\eta_{R} u\right|^{2} v+C e^{\lambda t} \int_{R \leq|x| \leq 2 R}\left(|u|^{2}+|\nabla u|^{2}\right) e^{-\lambda x_{n}} d x \\
\leq C \int_{\mathbf{R}^{n}}\left|\nabla\left(\eta_{R} u\right)\right|^{2}+C e^{\lambda t} \int_{R \leq|x| \leq 2 R}\left(|u|^{2}+|\nabla u|^{2}\right) e^{-\lambda x_{n}} d x .
\end{gathered}
$$

Letting $R \rightarrow \infty$ and using Lemma (1.6)(B) together with the hypothesis on $u$, we get

$$
\begin{aligned}
\int_{S_{t}}|u|^{2} e^{\lambda\left(t-x_{n}\right)} v d x & \leq C \lim _{R \rightarrow \infty} \int_{\mathbf{R}^{n}}\left(\left|\nabla \eta_{R}\right|^{2}|u|^{2}+\eta_{R}^{2}|\nabla u|^{2}\right) d x \\
& \leq C \int_{\mathbf{R}^{n}}|\nabla u|^{2} d x<\infty .
\end{aligned}
$$

Letting $\lambda \rightarrow \infty$, we see that $u \equiv 0$ in $S_{t}$.

We wish to point out another form of Lemma (2.1), helpful in other applications, which can be deduced from it by applying an affine transformation to $Q(D)$ as in [KRS]. 
Lemma (2.22). Let $z \in \mathbf{C}^{n}, \gamma \in \mathbf{C}$. Let $Q(D)=\Delta+z \cdot \nabla+\gamma$. Let $v \geq 0$, $v \in F_{p}, p>(n-1) / 2$ and $f \in C_{0}^{\infty}\left(\mathbf{R}^{n}\right)$. Then

$$
\int_{\mathbf{R}^{n}}|f|^{2} v d x \leq C \int_{\mathbf{R}^{n}}|Q(D) f|^{2} v^{-1} d x
$$

where $C$ does not depend on $f, \gamma$ or $z$.

\section{Proof of Theorem (1.2)}

Theorem (1.2) will be proved as usual using a Carleman inequality, but with a weighted $L^{2}\left(v^{-1}\right)$ to $L^{2}(v)$ estimate in place of the unweighted (except for powers) $L^{p}-L^{q}$ estimates of $[\mathrm{JK}]$.

Lemma (3.1). Suppose $v \in F_{p}$ for some $p>(n-1) / 2$. For every $\varepsilon$ with $0<\varepsilon<2 p-(n-1)$, there are $C, \beta>0$ depending only on $p, \varepsilon$ and $n$ such that

$$
\int_{\mathbf{R}^{n}} \frac{|u(x)|^{2}}{|x|^{2 t_{m}+n \pm 2}} v(x) d x \leq C\|v\|_{F_{p}}^{\beta} \int_{\mathbf{R}^{n}} \frac{|\Delta u(x)|^{2}}{|x|^{2 t_{m}+n \pm 2}} \frac{d x}{v(x)}
$$

for all $u \in C_{c}^{\infty}\left(\mathbf{R}^{n} \backslash\{0\}\right)$ and all $m \in \mathbf{Z}$, where $t_{m}=m-1+(1-\varepsilon) / 2$ if $m \geq 0$ and $t_{m}=m-1+(1+\varepsilon) / 2$ if $m \leq-1$, and the \pm sign is chosen according to the sign of $m:-$ if $m \geq 0$ and + if $m \leq-1$.

The case $t_{m} \rightarrow \infty$ in Lemma (3.1) will be used to prove part (A) of Theorem (1.3) while the case $t_{m} \rightarrow-\infty$ is used for part (B). We remark that the corresponding Carleman inequalities in [JK] have analogues for $t<0$, but these do not seem to have appeared in the literature.

To prove the Carleman estimate (3.2), we use E. Stein's complex interpolation $\left[\mathrm{ST}_{2}\right]$ on an analytic family of operators, essentially $(-\Delta)^{z / 2}$ minus a Taylor polynomial at the origin, as in $[\mathrm{JK}]$ but with two differences: we conjugate $(-\Delta)^{z / 2}$ with a complex power of the weight $v(x)$ and in the case $t<0$, we modify the kernel of $(-\Delta)^{z / 2}$ so as to be $C^{\infty}$ at infinity and then subtract a Taylor polynomial at infinity. We now describe the analytic family in detail. Let $\phi_{z}(x)=C_{z}|x|^{z-n}$ so that $\hat{\phi}_{z}(\xi)=(2 \pi|\xi|)^{-z}$. Let $m \in \mathbf{Z}$ and $m-1<t<m$. In the case $m \geq 0$, define

$$
\begin{aligned}
S_{z}^{t} g(x)=\frac{v(x)^{z / 4}}{\Gamma((n-z) / 2)} \int_{\mathbf{R}^{n}}\left[\phi_{z}(x-y)-\right. & \left.\left.\sum_{l=0}^{m-1} \frac{1}{l !}\left(\frac{\partial}{\partial s}\right)^{l} \phi_{z}(s x-y)\right|_{s=0}\right] \\
& \times\left(\frac{|y|}{|x|}\right)^{t+(n-z) / 2} v(y)^{z / 4} g(y) d y
\end{aligned}
$$

(where for $m=0$, the sum is empty) and in the case $m \leq-1$, define

$$
\begin{aligned}
& S_{z}^{t} g(x)=\frac{v(x)^{z / 4}}{\Gamma((n-z) / 2)} \int_{\mathbf{R}^{n}}|x|^{z-2} \\
& \quad \times\left[\psi_{z}(x, y)-\left.\sum_{l=0}^{|m|-2} \frac{1}{l !}\left(\frac{\partial}{\partial s}\right)^{l} \psi_{z}\left(s^{-1} x, y\right)\right|_{s=0}\right]\left(\frac{|y|}{|x|}\right)^{t+(n+z) / 2} v(y)=/ 4 g(y) d y
\end{aligned}
$$


where $\psi_{z}(x, y)=|x|^{2-z} \phi_{z}(x-y)$. Note that both $s \mapsto \phi_{z}(s x-y)$ and $s \mapsto$ $\psi_{z}\left(s^{-1} x, y\right)=|s|^{n-2}|x|^{2-z} \phi_{z}(x-s y)$ are $C^{\infty}$ near $s=0$ if $x, y \neq 0$-if $n$ is odd, we restrict $s$ to be nonnegative in $\psi_{z}\left(s^{-1} x, y\right)$ and interpret the symbol $\left.f(s)\right|_{s=0}$ appearing above to mean $\lim _{s \rightarrow 0^{+}} f(s)$. Now the family of operators $\left\{S_{z}^{t}\right\}_{0 \leq \operatorname{Re} z \leq n}$ is analytic in $z$ and $S_{2}^{t}$ satisfies the identity

$$
\frac{u(x) v(x)^{1 / 2}}{|x|^{t \pm 1+n / 2}}=S_{2}^{t}\left(\frac{\Delta u(y)}{v(y)^{1 / 2}|y|^{t \pm 1+n / 2}}\right)(x), \quad t \in \mathbf{R} \backslash \mathbf{Z},
$$

whenever $u \in C_{c}^{\infty}\left(\mathbf{R}^{n} \backslash\{0\}\right)$, and where \pm is chosen according to the sign of $m$. Thus Lemma (3.1) is equivalent to the boundedness of $S_{2}^{t}$ on $L^{2}\left(\mathbf{R}^{n}\right)$, and this in turn follows by complex interpolation $\left[\mathrm{ST}_{2}\right]$ once we have shown the boundedness of $S_{i \gamma}^{t}$ on $L^{2}\left(\mathbf{R}^{n}\right)$ (which for $t>0$ is Lemma 2.3 of [JK]) and, under the hypothesis $v \in F_{p}, p>(n-1) / 2$, the boundedness of $S_{n-1+\varepsilon+i \gamma}^{t}$ on $L^{2}\left(\mathbf{R}^{n}\right)$, for $0<\varepsilon / 2<p-(n-1) / 2$, with norms in both cases dominated by $C e^{c|\eta|}$.

First we prove that

$$
\int_{\mathbf{R}^{n}}\left|S_{i \gamma}^{t} f(x)\right|^{2} d x \leq C e^{c|\gamma|} \int_{\mathbf{R}^{n}}|f(x)|^{2} d x
$$

for all $f \in L^{2}\left(\mathbf{R}^{n}\right)$, where $C$ depends only on $\delta=\operatorname{distance}(t, \mathbf{Z})$ and $n$. To this end we introduce the family of operators $\left\{T_{z}^{t}\right\}_{0 \leq \operatorname{Re} z \leq 1}$ given by

$$
\begin{array}{r}
T_{z}^{t} g(x)=\frac{1}{\Gamma((n-z) / 2)} \int_{\mathbf{R}^{n}}\left[\phi_{z}(x-y)-\left.\sum_{l=0}^{m-1} \frac{1}{l !}\left(\frac{\partial}{\partial s}\right)^{l} \phi_{z}(s x-y)\right|_{s=0}\right] \\
\times\left(\frac{|y|}{|x|}\right)^{t}|y|^{-z} g(y) d y
\end{array}
$$

for $m-1<t<m, m \in \mathbf{Z}, m \geq 0$ and by

$$
\begin{array}{r}
T_{z}^{t} g(x)=\frac{1}{\Gamma((n-z) / 2)} \int_{\mathbf{R}^{n}}\left[\psi_{z}(x, y)-\left.\sum_{l=0}^{|m|-2} \frac{1}{l !}\left(\frac{\partial}{\partial s}\right)^{l} \psi_{z}\left(s^{-1} x, y\right)\right|_{s=0}\right] \\
\times\left(\frac{|y|}{|x|}\right)^{t+2-z}|y|^{-2} g(y) d y
\end{array}
$$

for $m-1<t<m, m \in \mathbf{Z}, m \leq 0$. Note that for $m=0$, the two definitions are identical and that for $m \geq 0, T_{z}^{t}$ coincides with the family of operators $T_{z}$ in [JK]. Clearly (3.3) is equivalent to

$$
\int_{\mathbf{R}^{n}}\left(\left.T_{i,}^{l} g(x)\right|^{2} \frac{d x}{|x|^{n}} \leq C e^{c|\eta|} \int_{\mathbf{R}^{n}}|g(x)|^{2} \frac{d x}{|x|^{n}}\right.
$$

for all $g \in L^{2}\left(|x|^{-n} d x\right)$, where $C$ depends only on $\delta$ and $n$. Since the case $m \geq 0$ of (3.4) is Lemma 2.3 of [JK], we suppose $m \leq 0$. As in [JK] we prove 
(3.4) by calculating the action of $T_{z}^{t}$ on spherical harmonics for $0<\operatorname{Re} z<1$, computing the Mellin transform and then letting $\operatorname{Re} z \rightarrow 0^{+}$. The details are similar to those in $[\mathrm{JK}]$, so we will refer liberally to that paper for the sake of brevity.

Let $g(r \omega)=h(r) P_{k}(\omega)$, where $r>0, \omega \in S^{n-1}, h \in C_{c}^{\infty}\left(\mathbf{R}_{+} \backslash\{0\}\right)$ and $P_{k}$ is a spherical harmonic homogeneous of degree $k$. Then according to Lemma 3.2 of [JK], if $-1<t<0$ and $\operatorname{Re} z>0$,

$$
T_{z}^{t} g(r \omega)=\int_{0}^{\infty} L^{\prime}\left(k, z, \frac{r}{s}\right)\left(\frac{r}{s}\right)^{z-t-2} h(s) \frac{d s}{s} P_{k}(\omega)
$$

where

$$
L^{\prime}(k, z, u)= \begin{cases}d(\nu, z) u^{k+2-z} F\left(\nu-z / 2,1-z / 2 ; \nu ; u^{2}\right), & 0<u<1, \\ d(\nu, z) u^{-n-k+2} F\left(\nu-z / 2,1-z / 2 ; \nu ; u^{-2}\right), & 1<u<\infty,\end{cases}
$$

and

$$
d(\nu, z)=\frac{2^{1-z} \Gamma(\nu-z / 2)}{\Gamma(\nu) \Gamma(z / 2) \Gamma(n / 2-z / 2)},
$$

$\nu=k+n / 2$ and $F(a, b ; c ; x)$ denotes the hypergeometric function. (Note that $L^{\prime}(k, z, u)=u^{2-z} L(k, z, u)$, where $L$ is as on page 468 of [JK].) Now set $g_{1}(y)=|y|^{t-z} g(y)$ and $f_{1}(x)=|x|^{2-z}(-\Delta)^{-z / 2} g_{1}(x)$ so that

$$
T_{z}^{t} g(x)=\frac{1}{\Gamma(n / 2-z / 2)}|x|^{-t+z-2}\left[f_{1}(x)-\left.\sum_{l=0}^{|m|-2} \frac{1}{l !}\left(\frac{\partial}{\partial s}\right)^{l} f_{1}\left(s^{-1} x\right)\right|_{s=0}\right] .
$$

Using (3.5), it follows that

$$
T_{z}^{t} g(r \omega)=\int_{0}^{\infty} L_{m}^{\prime}\left(k, z, \frac{r}{s}\right)\left(\frac{r}{s}\right)^{z-t-2} h(s) \frac{d s}{s} P_{k}(\omega),
$$

where

$$
L_{m}^{\prime}(k, z, u)=L^{\prime}(k, z, u)-\left.\sum_{l=0}^{|m|-2} \frac{1}{l !}\left(\frac{\partial}{\partial s}\right)^{l} L^{\prime}\left(k, z, s^{-1} u\right)\right|_{s=0} .
$$

Now let $R_{k}^{\prime}(u)=L_{m}^{\prime}(k, z, u) u^{-z-t-2}$. From the estimates for the hypergeometric function given in Remark 3.1 of [JK], it follows that $R_{k}^{\prime} \in L^{1}(d u / u)$ for $0<\operatorname{Re} z<t-(m-1)$. Indeed, the only singularities of $R_{k}^{\prime}$ occur at $u=0,1$, and $\infty$. The singularity at $u=1$ is integrable for $\operatorname{Re} z>0$ as in [JK] while $\left|R_{k}^{\prime}(u)\right| \leq C u^{-(|m|-2)} u^{\operatorname{Re} z-t-2}$ for $0 \leq u \leq \frac{1}{2}$ and $\left|R_{k}^{\prime}(u)\right| \leq$ $C u^{-(|m|-2)} u^{\operatorname{Re} z-t-2}$ for $2 \leq u<\infty$. Thus $R_{k}^{\prime}$ is integrable at 0 and $\infty$ provided $-(|m|-2)+\operatorname{Re} z-t-2>0$ and $-(|m|-1)+\operatorname{Re} z-t-2<0$ for $m \leq 0$, i.e., provided $m-1+\operatorname{Re} z<t<m+\operatorname{Re} z$, and in particular if $0<\operatorname{Re} z<t-(m-1)$. 
We now show that the formula for the Mellin transform of $R_{k}$ given in [JK], $m \geq 0$, remains valid for $R_{k}^{\prime}, m \leq 0$. According to (3.5) of [JK] (recall that $\left.L^{\prime}=u^{2-z} L\right)$,

$$
\begin{aligned}
2^{z} \int_{0}^{\infty} L^{\prime}(k, z, u) u^{-\lambda} \frac{d u}{u}=2^{z} \int_{0}^{\infty} L(k, z, u) u^{-(\lambda+z-2)} \frac{d u}{u} \\
\quad=\frac{\Gamma((k-\lambda-z+2) / 2) \Gamma((n+k-2+\lambda) / 2)}{\Gamma((k-\lambda+2) / 2) \Gamma((n+k+\lambda+z-2) / 2) \Gamma((n-z) / 2)}
\end{aligned}
$$

for $\operatorname{Re} z>0$ and $-\operatorname{Re}(k+n-2)<\operatorname{Re} \lambda<k-\operatorname{Re} z+2$. Now, in analogy with Lemma 3.4 of $[\mathrm{JK}]$, let $F(\lambda)$ denote $2^{-z}$ times the quotient of gamma functions above so that

$$
F(\lambda)=\int_{0}^{\infty} L^{\prime}(k, z, u) u^{-\lambda} \frac{d u}{u} \text { for } 0<\operatorname{Re} \lambda<2-\operatorname{Re} z .
$$

Suppose $L^{\prime}(k, z, u)=\sum_{l=0}^{\infty} c_{l} u^{-l}$ for $u$ large. Then, arguing as in [GS], for $m \leq-1,0<\operatorname{Re} z<1$, and $0<\operatorname{Re} \lambda<2-\operatorname{Re} z$,

$$
\begin{aligned}
F(\lambda)= & F(\lambda)-\int_{1}^{\infty} \sum_{l=0}^{|m|-2} c_{l} u^{-l-\lambda} \frac{d u}{u}+\sum_{l=0}^{|m|-2} \frac{c_{l}}{l+\lambda} \\
= & \int_{0}^{\infty} L^{\prime}(k, z, u) u^{-\lambda} \frac{d u}{u} \\
& -\int_{1}^{\infty}\left[\left.\sum_{l=0}^{|m|-2} \frac{1}{l !}\left(\frac{\partial}{\partial s}\right)^{l} L^{\prime}\left(k, z, s^{-1} u\right)\right|_{s=0}\right] u^{-\lambda} \frac{d u}{u}+\sum_{l=0}^{|m|-2} \frac{c_{l}}{l+\lambda} \\
= & \int_{0}^{1} L^{\prime}(k, z, u) u^{-\lambda} \frac{d u}{u}+\int_{1}^{\infty} L_{m}^{\prime}(k, z, u) u^{-\lambda} \frac{d u}{u}+\sum_{l=0}^{|m|-2} \frac{c_{l}}{l+\lambda} .
\end{aligned}
$$

However, the right-hand side above is meromorphic in $m+1<\operatorname{Re} \lambda<2-\operatorname{Re} z$ and so must coincide with $F(\lambda)$ in that region. On the other hand, if $\operatorname{Re} \lambda<$ $m+2$, then

$$
\begin{aligned}
\int_{0}^{1} L^{\prime}(k, z, u) u^{-\lambda} \frac{d u}{u}+\sum_{l=0}^{|m|-2} \frac{c_{l}}{l+\lambda} \\
\quad=\int_{0}^{1} L^{\prime}(k, z, u) u^{-\lambda} \frac{d u}{u}-\int_{0}^{1} \sum_{l=0}^{|m|-2} c_{l} u^{-l-\lambda} \frac{d u}{u} \\
\quad=\int_{0}^{1} L_{m}^{\prime}(k, z, u) u^{-\lambda} \frac{d u}{u}
\end{aligned}
$$

and combining these identities yields

$$
F(\lambda)=\int_{0}^{\infty} L_{m}^{\prime}(k, z, u) u^{-\lambda} \frac{d u}{u}, \quad \text { for } m+1<\operatorname{Re} \lambda<m+2 .
$$


Thus the Mellin transform of $R_{k}^{\prime}$ is given by

$$
\begin{aligned}
\widetilde{R}_{k}^{\prime}(\eta) & =\int_{0}^{\infty} R_{k}^{\prime}(u) u^{-i \eta} \frac{d u}{u}=\int_{0}^{\infty} L_{m}^{\prime}(k, z, u) u^{-(t+2-z+i \eta)} \frac{d u}{u} \\
& =\frac{\Gamma((k-t-i \eta) / 2) \Gamma((n+k+t-z+i \eta) / 2) 2^{-z}}{\Gamma((k-t+z-i \eta) / 2) \Gamma((n+k+t+i \eta) / 2) \Gamma((n-z) / 2)}
\end{aligned}
$$

for $0<\operatorname{Re} z<1$ and $m-1+\operatorname{Re} z<t<m+\operatorname{Re} z, m \leq 0$.

We now complete the proof of (3.4) as in [JK] by expanding $g$ in spherical harmonics as follows:

$$
g(r \omega)=\sum_{\substack{k \geq 0 \\ 1 \leq l \leq a_{k}}} g_{k, l}(r) P_{k, l}(\omega),
$$

where $g_{k, l} \in C_{c}^{\infty}\left(\mathbf{R}_{+} \backslash\{0\}\right)$ and $\left\{P_{k, l}\right\}_{l=1}^{a_{k}}$ is an orthonormal basis for the vector space of spherical harmonics of degree $k$. If we set

$$
T_{z, k}^{t}(h)(r)=\int_{0}^{\infty} R_{k}^{\prime}\left(\frac{r}{s}\right) h(s) \frac{d s}{s}
$$

then

$$
T_{i \gamma} g(x)=\lim _{\varepsilon \rightarrow 0} T_{\varepsilon+i \gamma} g(x)=\lim _{\varepsilon \rightarrow 0} \sum_{k, l} T_{\varepsilon+i \gamma, k}\left(g_{k, l}\right)(r) P_{k, l}(\omega)
$$

and it follows from (3.6), (3.7) and Plancherel's theorem that

$$
\begin{aligned}
& \int_{\mathbf{R}^{n}}\left|T_{i \gamma} g(x)\right|^{2} \frac{d x}{|x|^{n}} \leq \lim _{\varepsilon \rightarrow 0} \sum_{k, l} \int_{0}^{\infty}\left|T_{\varepsilon+i \gamma, k}\left(g_{k, l}\right)(r)\right|^{2} \frac{d r}{r} \\
& \quad=\lim _{\varepsilon \rightarrow 0} \sum_{k, l} \int_{-\infty}^{\infty}\left|\frac{2^{-(\varepsilon+i \eta)} \Gamma((k-t-i \eta) / 2) \Gamma((n+k+t-\varepsilon-i \gamma+i \eta) / 2)}{\Gamma((n-\varepsilon-i \gamma) / 2) \Gamma((k-t+\varepsilon+i \gamma-i \eta) / 2) \Gamma((n+k+t+i \eta) / 2)}\right|\left|\tilde{g}_{k, l}(\eta)\right|^{2} d \eta .
\end{aligned}
$$

Using Stirling's formula as in [JK], the above is dominated by

$$
\begin{aligned}
C e^{c|\gamma|} \sum_{k, l} \int_{-\infty}^{\infty}\left|\tilde{g}_{k, l}(\eta)\right|^{2} d \eta & =C e^{c|\gamma|} \sum_{k, l} \int_{0}^{\infty}\left|g_{k, l}(r)\right|^{2} \frac{d r}{r} \\
& =C e^{c|\gamma|} \int_{\mathbf{R}^{n}}|g(x)|^{2} \frac{d x}{|x|^{n}} .
\end{aligned}
$$

This completes the proof of (3.4) and (3.3).

To complete the proof of Lemma (3.1) it remains only to prove, under the hypothesis $v \in F_{p}, p>(n-1) / 2$, the boundedness of the operator $S_{n-1+\varepsilon+i \gamma}^{t}$ on $L^{2}\left(\mathbf{R}^{n}\right), 0<\varepsilon / 2<p-(n-1) / 2$, with norm dominated by $C e^{c|\gamma|}$. The key here is the following inequality of $\mathrm{E}$. Stein $\left[\mathrm{St}_{1}\right]$ (cf. [Sa]):

$$
\begin{gathered}
\left|1-r e^{i \theta}\right|^{-2 \lambda}-\left.\sum_{l=0}^{m-1} \frac{1}{l !}\left(\frac{\partial}{\partial s}\right)^{l}\left|1-s r e^{i \theta}\right|^{-2 \lambda}\right|_{s=0} \mid \\
\leq C_{\lambda} r^{m-1+2 \operatorname{Re} \lambda}\left|1-r e^{i \theta}\right|^{-2 \operatorname{Re} \lambda}
\end{gathered}
$$


for $r>0, \theta \in \mathbf{R}, m \in \mathbf{Z}_{+}$and $0<\operatorname{Re} \lambda<\frac{1}{2}$ and where the constant $C_{\lambda}$ is independent of $m, r, \theta$ and satisfies $C_{\lambda} \leq C_{\mu} e^{c_{\mu}|\nu|}, \lambda=\mu+i \nu$. Fix $x, y \in \mathbf{R}^{n}$ momentarily and identify the points $0, y /|y|, x /|y|$ in $\mathbf{R}^{n}$ with the numbers $0,1, r e^{i \theta}$ in the complex plane. Since

$$
\phi_{z}(s x-y)=c_{z}|y|^{z-n}\left|\frac{y}{|y|}-s \frac{x}{|y|}\right|^{z-n},
$$

(3.8) becomes with $2 \lambda=n-z$,

$$
\begin{aligned}
& \left|\phi_{z}(x-y)-\sum_{l=0}^{m-1} \frac{1}{l !}\left(\frac{\partial}{\partial s}\right)^{l} \phi_{z}(s x-y)\right|_{s=0} \mid \\
& \quad \leq C_{z}\left(\frac{|x|}{|y|}\right)^{m-1+n-\operatorname{Re} z} \phi_{\operatorname{Re} z}(x-y), \quad 0<\operatorname{Re} \frac{n-z}{2}<\frac{1}{2} .
\end{aligned}
$$

Now identify $0, x /|x|, y /|x|$ with $0,1, r e^{i \theta}$. Since

$$
\psi_{z}\left(s^{-1} x, y\right)=c_{z} s^{n-2}|x|^{2-n}\left|\frac{x}{|x|}-s \frac{y}{|x|}\right|^{z-n},
$$

(3.8) becomes with $2 \lambda=n-z$,

$$
\begin{aligned}
\left|\psi_{z}(x, y)-\sum_{l=0}^{|m|-2} \frac{1}{l !}\left(\frac{\partial}{\partial s}\right)^{l} \psi_{z}\left(s^{-1} x, y\right)\right| & \\
\leq C_{z}\left(\frac{|y|}{|x|}\right)^{|m|-\operatorname{Re} z} \psi_{z}(x, y), \quad 0 & <\operatorname{Re} \frac{n-z}{2}<\frac{1}{2} .
\end{aligned}
$$

(Note that in obtaining (3.10), we use (3.8) with $m$ replaced by $|m|-2-(n-2)$.) With $t_{m}=m-1+(1-\varepsilon) / 2$ in case $m \geq 0$ and $t_{m}=m-1+(1+\varepsilon) / 2$ in case $m \leq-1$, we obtain from (3.9) and (3.10) that

$$
\left|S_{n-1+\varepsilon+i \gamma}^{t_{m}} g(x)\right| \leq C_{\gamma} v(x)^{(n-1+\varepsilon) / 4} \int_{\mathbf{R}^{n}} \phi_{n-1+\varepsilon}(x-y) v(y)^{(n-1+\varepsilon) / 4}|g(y)| d y
$$

for $m \in \mathbf{Z}$ with $C_{\gamma}$ independent of $m$. Thus the boundedness of $S_{n-1+\varepsilon+i \gamma}^{t_{m}}$ on $L^{2}$ with norm dominated by $C e^{c|\gamma|}$ will follow from the inequality

$$
\int_{\mathbf{R}^{n}}\left(I_{n-1+\varepsilon} f\right)^{2} v^{(n-1+\varepsilon) / 2} \leq C \int_{\mathbf{R}^{n}} f^{2} v^{-(n-1+\varepsilon) / 2}, \quad f \geq 0 .
$$

However, if $v \in F_{p}, p>(n-1+\varepsilon) / 2$, then (3.11) holds with $C=C_{p, n, \varepsilon}$ by [CW], since we can replace $v$ by $v^{*}=M\left(v^{q}\right)(x)^{1 / q}, p>q>(n-1+\varepsilon) / 2$, as in the proof of Lemma (2.2) in $\S 2$. Alternatively, (3.11) is equivalent to the trace inequality

$$
\int_{\mathbf{R}^{n}}\left(I_{(n-1+\varepsilon) / 2} f\right)^{2} v^{(n-1+\varepsilon) / 2} \leq C \int_{\mathbf{R}^{n}} f^{2}, \quad f \geq 0 .
$$

(To see this, simply compose (3.12) with its dual inequality,

$$
\int\left(I_{(n-1+\varepsilon) / 2} f\right)^{2} \leq C \int_{\mathbf{R}^{n}} f^{2} v^{-(n-1+\varepsilon) / 2},
$$


and use $\left.I_{\alpha}\left(I_{\alpha} f\right)=I_{2 \alpha} f.\right)$ See [F] and [KS] for the relevant results on (3.12). This completes the proof of the Carleman inequality, Lemma (3.1).

Proof of Theorem (1.2). (A) As in [JK], it suffices to show that if $u$ vanishes to infinite order at $a$ and satisfies the differential inequality $|\Delta u| \leq v|u|$, then $u$ vanishes identically in $B(a, R)$ whenever $\left\|\chi_{B(a, R)} v\right\|_{F_{p}}<\varepsilon(p, n)$. Here $B(a, R)=\{x:|x-a|<R\}$ if $a \in \mathbf{R}^{n}$ and $B(\infty, R)=\{x:|x|>R\}$ if $a=\infty$. Furthermore, we may assume $v \geq 1$ since $v+1$ also satisfies the hypotheses of Theorem (1.3). We begin with the case $a \in \mathbf{R}^{n}$ and suppose without loss of generality that $a=0$. Momentarily fix $R>0$ and let $\eta \in C_{c}^{\infty}(B(0,2 R))$ satisfy $\eta=1$ on $B(0, R)$. In addition, let $\psi \in C^{\infty}\left(\mathbf{R}^{n}\right)$ satisfy $\psi=0$ on $B(0,1), \psi=1$ outside $B(0,2), 0 \leq \psi \leq 1$, and set $\psi_{k}(x)=\psi(k x)$ for $k \geq 1$. Then for $k \geq 4 / R$ and $m \geq 0$, Lemma (3.1) gives

$$
\begin{aligned}
\int_{|x|<R} & \frac{\left|\psi_{k}(x) u(x)\right|^{2}}{|x|^{2 t_{m}+n-2}} v(x) d x \\
\leq & C_{p, n}\left\|\chi_{B(0, R)} v\right\|_{F_{p}}^{\beta} \int_{\mathbf{R}^{n}} \frac{\left|\Delta\left(\psi_{k} \eta u\right)(x)\right|^{2}}{|x|^{2 t_{m}+n-2}} \frac{d x}{v(x)} \\
\leq & C_{p, n}\left\|\chi_{B(0, R)} v\right\|_{F_{p}}^{\beta}\left[\int_{\mathbf{R}^{n}} \frac{\left|\Delta \psi_{k}(x)\right|^{2}|u(x)|^{2}}{|x|^{2 t_{m}+n-2}} \frac{d x}{v(x)}\right. \\
& +\int_{\mathbf{R}^{n}} \frac{\left|\nabla \psi_{k}(x)\right|^{2}|\nabla u(x)|^{2}}{|x|^{2 t_{m}+n-2}} \frac{d x}{v(x)} \\
& \left.+\left(\int_{|x|<R}+\int_{|x|>R}\right) \frac{\left|\psi_{k}(x)\right|^{2}|\Delta(\eta u)(x)|^{2}}{|x|^{2 t_{m}+n-2}} \frac{d x}{v(x)}\right] \\
= & \mathrm{I}_{k}+\mathrm{II}_{k}+\mathrm{III}_{k}+\mathrm{IV}_{k} \cdot \quad .
\end{aligned}
$$

If $\left\|\chi_{B(0, R)} v\right\|_{F_{p}}<\varepsilon(p, n)$, where $\varepsilon(p, n)$ is chosen so that $C_{p, n} \varepsilon(p, n)^{\beta}=\frac{1}{2}$, then III $_{k}$ is at most one-half the left side of (3.13) and subtracting III $_{k}$ from both sides of (3.13) we obtain

$$
\int_{|x|<R} \frac{\left|\psi_{k}(x)\right|^{2}|u(x)|^{2}}{|x|^{2 t_{m}+n-2}} v(x) d x \leq 2\left(\mathrm{I}_{k}+\mathrm{II}_{k}+\mathrm{IV}_{k}\right) .
$$

However, letting $k \rightarrow \infty$ we get

$$
\int_{|x|<R} \frac{|u(x)|^{2}}{|x|^{2 t_{m}+n-2}} v(x) d x \leq 2 \int_{|x|>R} \frac{|\Delta(\eta u)(x)|^{2}}{|x|^{2 t_{m}+n-2}} \frac{d x}{v(x)}, \quad m \geq 0
$$

since $\lim _{k \rightarrow \infty} I_{k}=\lim _{k \rightarrow \infty} I_{k}=0$ (whose proof we give in a moment). Now multiply both sides of $(3.14)$ by $R^{2 t_{m}+n-2}$ and then let $m \rightarrow \infty$ to conclude 
that $|u|^{2} v$, and hence $u$ is identically zero on $B(0, R)$. It remains only to show $\lim _{k \rightarrow \infty} \mathrm{I}_{k}=\lim _{k \rightarrow \infty} \mathrm{II}_{k}=0$. But

$$
\mathbf{I}_{k}=\int_{1 / k \leq|x| \leq 2 / k} \frac{\left|\Delta \psi_{k}(x)\right|^{2}|u(x)|^{2}}{|x|^{2 t_{m}+n-2}} \frac{d x}{v(x)} \leq C k^{2 t_{m}+n+2} \int_{|x| \leq 2 / k}|u(x)|^{2} d x,
$$

which tends to zero as $k \rightarrow \infty$ since $u$ vanishes to infinite order at 0 . Similarly

$$
\begin{aligned}
\mathrm{II}_{k} \leq C k^{2 t_{m}+n} & \int_{|x| \leq 2 / k}|\nabla u(x)|^{2} d x \\
\leq C k^{2 t_{m}+n}\left[\left(\int_{|x| \leq 4 / k}|u(x)|^{2} d x\right)^{1 / 2}\right. & \left(\int_{|x| \leq 4 / k}|\Delta u(x)|^{2} d x\right)^{1 / 2} \\
& \left.+k^{2} \int_{|x| \leq 4 / k}|u(x)|^{2} d x\right]
\end{aligned}
$$

by Lemma (1.6)(A) and this last term tends to zero as $k \rightarrow \infty$ since $u$ vanishes to infinite order at 0 and $\Delta u \in L_{\text {loc }}^{2}$.

Proof of Theorem (1.2). (B) In the case $u$ vanishes to infinite order at infinity, we argue as in the proof of part (A) above, but with $\eta \in C^{\infty}$ such that $\eta=1$ on $B(\infty, R)=\{x:|x|>R\}$ and $\eta=0$ on $B(0, R / 2) ; \psi \in C^{\infty}$ such that $\psi=0$ on $B(\infty, 1)$ and $\psi=1$ on $B\left(0, \frac{1}{2}\right) ; \psi_{k}(x)=\psi(x / k)$. Furthermore, we may assume that $v \geq \varepsilon|x|^{-2}$ since $v+\varepsilon|x|^{-2}$ also satisfies the hypothesis of Theorem (1.2)(B). The result is that (3.14) becomes

$$
\int_{|x|>R} \frac{|u(x)|^{2}}{|x|^{2 t_{m}+n+2}} v(x) d x \leq \int_{|x|<R} \frac{|\Delta(\eta u)(x)|^{2}}{|x|^{2 t_{m}+n+2}} \frac{d x}{v(x)}, \quad m \leq-1 .
$$

Note that the hypothesis $\int_{\mathbf{R}^{n}}|\Delta u|^{2}<\infty$ is needed to show that the term corresponding to $\mathrm{II}_{k}$ in (3.13) tends to zero as $k$ tends to infinity. Now multiply both sides by $R^{2 t_{m}+n+2}$ and then let $m \rightarrow-\infty$ to conclude that $u \equiv 0$ in $\{x:|x|>R\}$. This completes the proof of Theorem (1.2).

\section{UNIQUE CONTINUATION IN LOW DIMENSIONS}

We begin by proving Theorem (1.4). Recall that by the definition of \|\|$v\|\|$, we have

$$
\int_{\mathbf{R}^{n}}\left|I_{1} f(x)\right|^{2} v(x) d x \leq\left\|\left.|| v\left|\| \int_{\mathbf{R}^{n}}\right| f(x)\right|^{2} d x, \quad \text { for } f \geq 0,\right.
$$

where $I_{1}$ denotes the fractional integral of order one. If we compose this inequality with its dual, $\int\left|I_{1} f\right|^{2} \leq|||v| \| \int|f|^{2} v^{-1}$, we obtain the equivalent inequality (for the reverse implication, apply Hölder's inequality to $\int\left|I_{1} f\right|^{2}=$ $\left.\int f I_{2} f\right)$

$$
\int_{\mathbf{R}^{n}}\left|I_{2} f(x)\right|^{2} v(x) d x \leq\|\| v \|\left.\right|^{2} \int_{\mathbf{R}^{n}}|f(x)|^{2} \frac{d x}{v(x)}, \quad \text { for } f \geq 0 .
$$


We now prove Theorem (1.4) by using the following pointwise inequality [Sa], which essentially corresponds to the cases $\lambda=0$ and $\frac{1}{2}$ of (3.8):

$$
\left|\phi_{2}(x-y)-\sum_{l=0}^{m-1} \frac{1}{l !}\left(\frac{\partial}{\partial s}\right)^{l} \phi_{2}(s x-y)\right|_{s=0} \mid \leq C\left(\frac{|x|}{|y|}\right)^{m} \phi_{2}(x-y),
$$

for all $m \in \mathbf{Z}_{+}, x, y \in \mathbf{R}^{n}, n=2$ or 3 , and where $C$ is independent of $m, x$ and $y$. If $n=2$, we must restrict $|x|,|y|<\frac{1}{4}$.

Now suppose $u$ vanishes to infinite order at 0 and satisfies $|\Delta u| \leq v|u|$. With $\eta$ and $\psi_{k}$ as in $\S 3$, we have for $m \in \mathbf{Z}_{+}$,

$$
\begin{aligned}
\int_{|x|<R} & \frac{\left|\psi_{k}(x) u(x)\right|^{2}}{|x|^{2 m}} v(x) d x \\
= & \int_{|x|<R}|x|^{-2 m}\left|\int \phi_{2}(x-y) \Delta\left(\psi_{k} \eta u\right)(y) d y\right|^{2} v(x) d x \\
= & \int_{|x|<R}|x|^{-2 m} \\
& \times\left|\int\left[\phi_{2}(x-y)-\left.\sum_{l=0}^{m-1} \frac{1}{l !}\left(\frac{\partial}{\partial s}\right)^{l} \phi_{2}(s x-y)\right|_{s=0}\right] \Delta\left(\psi_{k} \eta u\right)(y) d y\right|^{2} v(x) d x \\
\leq & C^{2} \int_{|x|<R}\left(\int \phi_{2}(x-y) \frac{\left|\Delta\left(\psi_{k} \eta u\right)(y)\right|}{|y|^{m}} d y\right)^{2} v(x) d x \quad \text { by }(4.2) \\
\leq & C^{2}|| \chi_{B(0, R)} v \mid \|^{2} \int_{\mathbf{R}^{n}} \frac{\left|\Delta\left(\psi_{k} \eta u\right)(x)\right|^{2}}{|x|^{2 m}} \frac{d x}{v(x)} \text { by (4.1). }
\end{aligned}
$$

Provided $R$ is small enough that $C^{2}\left\|\chi_{B(0, R)} v\right\| \|^{2}<\frac{1}{2}$, we can now argue as in the proof of Theorem (1.3) and conclude that $u \equiv 0$ on $B(0, R)$. A standard connectedness argument then completes the proof of Theorem (1.4).

We now restrict attention to dimension $n=2$ and give the proof of Theorem (1.5). In order to handle the gradient term in the differential inequality $|\Delta u| \leq w|\nabla u|$, we use a pointwise inequality corresponding to (4.2) but with $\phi_{2}$ replaced by $R_{j} \phi_{1}$, where $R_{j}$ denotes the $j$ th Riesz transform, $j=1,2$ :

$$
\left|R_{j} \phi_{1}(x-y)-\sum_{l=0}^{m-1} \frac{1}{l !}\left(\frac{\partial}{\partial s}\right)^{l} R_{j} \phi_{1}(s x-y)\right|_{s=0} \mid \leq\left(\frac{|x|}{|y|}\right)^{m} \phi_{1}(x-y),
$$

for all $m \in \mathbf{Z}_{+}$and $x, y$ in $\mathbf{R}^{2}$. The proof of (4.3) is easy due to the especially simple form of $R_{j} \phi_{1}$;

$$
\left(R_{j} \phi_{1}\right)^{\wedge}(\xi)=\frac{\xi_{j}}{|\xi|} \hat{\phi}_{1}(\xi)=\frac{\xi_{j}}{|\xi|^{2}}
$$

implies $R_{j} \phi_{1}(x)=c x_{j} /|x|^{2} \quad(n=2)=c\left\langle x, e_{j}\right\rangle /|x|^{2}$, a rational function of $x$. 
To prove (4.3), we introduce the function

$$
\begin{aligned}
\psi_{\lambda}(z) & =\frac{e^{i \lambda} \operatorname{Re}\left(e^{-i \lambda}(1-z)\right)}{|1-z|^{2}}=\frac{(1-z)+e^{i 2 \lambda}(1-\bar{z})}{2|1-z|^{2}} \\
& =\frac{1}{2(1-\bar{z})}+\frac{e^{i 2 \lambda}}{2(1-z)}, \quad z \in \mathbf{C} .
\end{aligned}
$$

Then

$$
\begin{gathered}
\left|\psi_{\lambda}(z)-\sum_{l=0}^{m-1} \frac{1}{l !}\left(\frac{\partial}{\partial s}\right)^{l} \psi_{\lambda}(s z)\right|_{s=0}|=| \psi_{\lambda}(z)-\sum_{l=0}^{m-1} \frac{\bar{z}^{l}+e^{i 2 \lambda} z^{l}}{2} \mid \\
=\left|\frac{\bar{z}^{m}}{2(1-\bar{z})}+e^{i 2 \lambda} \frac{z^{m}}{2(1-z)}\right| \leq|z|^{m}|1-z|^{-1},
\end{gathered}
$$

for all $m \in \mathbf{Z}_{+}$. Now momentarily fix $x, y$ in $\mathbf{R}^{2}$ and, by rotating the plane, identify $y /|y|$ and $x /|y|$ with 1 and $z$ in the complex plane. Suppose that under this rotation, the unit vector $e_{j}$ in $\mathbf{R}^{2}$ is identified with $e^{i \lambda}$ in $\mathbf{C}$. Then

$$
R_{j} \phi_{1}(x-y)=-|y|^{-1} R_{j} \phi_{1}\left(\frac{y}{|y|}-\frac{x}{|y|}\right)=-|y|^{-1} e^{-i \lambda} \psi_{\lambda}(z)
$$

and (4.4) yields

$$
\begin{gathered}
\left.\left|R_{j} \phi_{1}(x-y)-\sum_{l=0}^{m-1} \frac{1}{l !}\left(\frac{\partial}{\partial s}\right)^{l} R_{j} \phi_{1}(s x-y)\right|_{s=0}|\leq| y\right|^{-1}|z|^{m}|1-z|^{-1} \\
=\left(\frac{|x|}{|y|}\right)^{m}|y|^{-1}\left|\frac{y}{|y|}-\frac{x}{|y|}\right|^{-1}=\left(\frac{|x|}{|y|}\right)^{m}|x-y|^{-1},
\end{gathered}
$$

which is $(4.3)$.

Now suppose $u$ vanishes to infinite order at 0 and satisfies the differential inequality $|\Delta u| \leq w|\nabla u|$. Let $\eta$ and $\psi_{k}$ be as in $\S 3$. We use the following weighted inequality (which follows from [CW] as in the proof of Lemma 1.2 in $\S 2$ above)

$$
\int\left(I_{1} g\right)^{2} w \leq C_{p}\left\|w^{2}\right\|_{F_{p / 2}} \int g^{2} w^{-1}, \quad \text { for } g \geq 0,
$$

for $p>1, n=2$, together with the identity

$$
\begin{aligned}
|\nabla f(x)|^{2} & =\sum_{j=1}^{2}\left|\int_{\mathbf{R}^{n}} R_{j} \phi_{1}(x-y) \Delta f(y) d y\right|^{2} \\
& =\sum_{j=1}^{2}\left|\int_{\mathbf{R}^{n}}\left[R_{j} \phi_{1}(x-y)-\left.\sum_{l=0}^{m-1} \frac{1}{l !}\left(\frac{\partial}{\partial s}\right)^{l} R_{j} \phi_{1}(s x-y)\right|_{s=0}\right] \Delta f(y) d y\right|^{2}
\end{aligned}
$$


for $f \in C_{c}^{\infty}\left(\mathbf{R}^{2} \backslash\{0\}\right)$, to obtain

$$
\begin{aligned}
& \int_{|x|<R} \frac{\left|\nabla\left(\psi_{k} u\right)(x)\right|^{2}}{|x|^{2 m}} w(x) d x \\
& =\sum_{j=1}^{2} \int_{|x|<R}|x|^{-2 m} \mid \int_{\mathbf{R}^{n}}\left[R_{j} \phi_{1}(x-y)-\sum_{l=0}^{m-1} \frac{1}{l !}\left(\frac{\partial}{\partial s}\right)^{l}\right. \\
& \left.\quad \times\left. R_{j} \phi_{1}(s x-y)\right|_{s=0}\right]\left.\Delta\left(\psi_{k} \eta u\right)(y) d y\right|^{2} w(x) d x, \\
& \leq 2 \int_{|x|<R}\left(\int_{\mathbf{R}^{n}} \phi_{1}(x-y) \frac{\left|\Delta\left(\psi_{k} \eta u\right)(y)\right|}{|y|^{m}} d y\right)^{2} w(x) d x, \quad \text { by }(4.3), \\
& \leq C_{p}\left\|\chi_{B(0, R)} w^{2}\right\|_{F_{p / 2}}^{1 / 2} \int_{\mathbf{R}^{n}} \frac{\left|\Delta\left(\psi_{k} \eta u\right)(x)\right|^{2}}{|x|^{2 m}} \frac{d x}{w(x)}, \quad \text { by }(4.5), \\
& \leq C_{p}\left\|\chi_{B(0, R)} w^{2}\right\|_{F_{p / 2}}^{1 / 2}\left[\int \frac{\left|\Delta \psi_{k}\right|^{2}|u|^{2}}{|x|^{2 m}} \frac{d x}{w(x)}+\int \frac{\left|\nabla \psi_{k}\right|^{2}|\nabla u|^{2}}{|x|^{2 m}} \frac{d x}{w(x)}\right. \\
& \left.+\left(\int_{|x|<R}+\int_{|x|>R}\right) \frac{\left|\psi_{k}\right|^{2}|\Delta(\eta u)|^{2}}{|x|^{2 m}} \frac{d x}{w(x)}\right]
\end{aligned}
$$

Now $\mathrm{I}_{k}, \mathrm{II}_{k}$, and $\mathrm{IV}_{k}$ can be handled just as in the proof (see (3.13)) of Theorem (1.3). From the differential inequality $|\Delta u| \leq w|\nabla u|$, we obtain

$$
\begin{aligned}
& \mathrm{III}_{k}=C_{p}\left\|\chi_{B(0, R)} w^{2}\right\|_{F_{p / 2}}^{1 / 2} \int_{|x|<R} \frac{\left|\psi_{k}\right|^{2}|\Delta u|^{2}}{|x|^{2 m}} \frac{d x}{w(x)} \\
& \leq C C_{p}\left\|\chi_{B(0, R)} w^{2}\right\|_{F_{p / 2}}^{1 / 2} \int_{|x|<R} \frac{\left|\psi_{k}\right|^{2}|\nabla u|^{2}}{|x|^{2 m}} w(x) d x \\
& \leq C C_{p}\left\|\chi_{B(0, R)} w^{2}\right\|_{F_{p / 2}}^{1 / 2}\left[\int_{|x|<R} \frac{\left|\nabla\left(\psi_{k} u\right)\right|^{2}}{|x|^{2 m}} w(x) d x\right. \\
&\left.\quad+\int_{|x|<R} \frac{\left|\nabla \psi_{k}\right|^{2}|u|^{2}}{|x|^{2 m}} w(x) d x\right] \\
&=\mathrm{V}_{k}+\mathrm{VI}_{k},
\end{aligned}
$$

since $\psi_{k} \nabla u=\nabla\left(\psi_{k} u\right)-\left(\nabla \psi_{k}\right) u$. Now if $R$ is so small that $C C_{p}\left\|\chi_{B(0, R)} w^{2}\right\|_{F_{p / 2}}^{1 / 2}$ $\leq \frac{1}{2}$, then $V_{k}$ is at most one-half the left side of (4.7) and so can be subtracted from both sides as in the proof (see (3.13)) of Theorem (1.3). It remains only 
to show that $\lim _{k \rightarrow \infty} \mathrm{VI}_{k}=0$. However, since $\nabla \psi_{k}$ is supported in $\{x: 1 / k \leq$ $|x| \leq 2 / k\}$,

$$
\begin{aligned}
\mathrm{VI}_{k} \leq C k^{2 m+2} \int_{|x| \leq 2 / k}|u(x)|^{2} w(x) d x & \\
\leq & \leq k^{2 m+2}\left[\int_{|x| \leq 2 / k}\left|u(x)-\frac{1}{B(0,2 / k)} \int_{B(0,2 / k)} u\right|^{2} w(x) d x\right. \\
& \left.+\left(\int_{B(0,2 / k)} w\right)\left(\frac{1}{B(0,2 / k)} \int_{B(0,2 / k)}|u|\right)^{2}\right] \\
& =\mathrm{VII}_{k}+\mathrm{VIII}_{k} .
\end{aligned}
$$

Now $\lim _{k \rightarrow \infty}$ VIII $_{k}=0$ since $u$ vanishes to infinite order at 0 and

$$
\begin{aligned}
\mathrm{VII}_{k} & \leq C k^{2 m+2} \int_{|x| \leq 2 / k}\left|I_{1}\left[\chi_{B(0,2 / k)}(y)|\nabla u(y)|\right](x)\right|^{2} w(x) d x \\
& \leq C k^{2 m+2} \int_{|x| \leq 2 / k}|\nabla u(x)|^{2} \frac{d x}{w(x)}, \quad \text { by (4.5). }
\end{aligned}
$$

This last expression tends to zero as $k$ tends to infinity by the argument used to handle term $\mathrm{II}_{k}$ in the proof of Theorem (1.3) in $\S 3$. This completes the proof of Theorem (1.5).

We remark that Theorem (1.5) can be sharpened in the spirit of Theorem (1.4) by replacing the quantity $\left\|w^{2}\right\|_{F_{p / 2}}, p>1$, in (4.5) with the norm of the embedding $I_{1 / 2}: L^{2} \rightarrow L^{2}(w)$.

\section{REFERENCES}

[BKRS] B. Barcelo, C. E. Kenig, A. Ruiz and C. D. Sogge, Weighted Sobolev inequalities and unique continuation for the Laplacian plus lower order terms, Preprint.

[CW] S. Chanillo and R. Wheeden, $L^{p}$ estimates for fractional integrals and Sobolev inequalities with applications to Schrodinger operators, Comm. Partial Differential Equations 10 (1985), 1077-1116.

[ChR] S. Chiarenza and A. Ruiz,

[CR] R. Coifman and R. Rochberg, Another characterization of BMO, Proc. Amer. Math. Soc. 79 (1980), 249-254.

[F] C. Fefferman, The uncertainty principle, Bull. Amer. Math. Soc. (N.S.) 9 (1983), 129-206.

[GS] I. Gelfand and G. Shilov, Generalized functions, Academic Press, 1964.

[JK] D. Jerison and C. E. Kenig, Unique continuation and absence of positive eigenvalues for Schrodinger operators, Ann. of Math. (2) (1985), 463-494.

[K] C. E. Kenig, Lecture notes at conference in harmonic analysis at El Escorial, 1987.

[KRS] C. E. Kenig, A. Ruiz and C. Sogge, Sobolev inequalities and unique continuation for second order constant coefficient differential equations, Duke Math. J. 55 (1987), 329-348.

[KS] R. Kerman and E. Sawyer, The trace inequality and eigenvalue estimates for Schrödinger operators, Ann. Inst. Fourier (Grenoble) 36 (1986), 207-228.

$[\mathrm{K}]$ D. Kurtz, Littlew'ood-Paley and multiplier theorems on weighted $L^{p}$ spaces, Trans. Amer. Math. Soc. 259 (1980), 235-254. 
[R] G. Roberts, Uniqueness in the Cauchy problem for characteristic operators of Fuchsian type, J. Differential Equations 38 (1980), 374-392.

[Sa] E. Sawyer, Unique continuation for Schrödinger operators in dimension three or less, Ann. Inst. Fourier (Grenoble) 34 (1984), 189-200.

[St $\left.{ }_{1}\right]$ E. Stein, Appendix to unique continuation, Ann. of Math. (2) 121 (1985), 489-494.

[St $\left.{ }_{2}\right]$ _ Interpolation of linear operators, Trans. Amer. Math. Soc. 83 (1956), 482-492.

[T] P. Tomas, Restriction theorems for the Fourier transform, Proc. Sympos. Pure Math., vol. 35, part 1, Amer. Math. Soc., Providence, R.I., pp. 111-114.

Department of Mathematics, Rutgers University, New Brunswick, New Jersey 08903

Department of Mathematics and Statistics, McMaster University, Hamilton, ONTARIO L8S 4K1 CANADA 